Article

\title{
Phytochemical Profiling of Coryphantha macromeris (Cactaceae) Growing in Greenhouse Conditions Using Ultra-High-Performance Liquid Chromatography-Tandem Mass Spectrometry
}

\author{
Emmanuel Cabañas-García ${ }^{1}$, Carlos Areche ${ }^{2}$, Juan Jáuregui-Rincón ${ }^{1}(1)$, \\ Francisco Cruz-Sosa ${ }^{3, *(D)}$ and Eugenio Pérez-Molphe Balch ${ }^{1}$ \\ 1 Centro de Ciencias Básicas, Universidad Autónoma de Aguascalientes, Av. Universidad 940, \\ 20131 Aguascalientes, Mexico; fde_garci@hotmail.com (E.C.-G.); jjaureg@correo.uaa.mx (J.J.-R.); \\ eperezmb@correo.uaa.mx (E.P.-M.B.) \\ 2 Departamento de Química, Facultad de Ciencias, Universidad de Chile, Casilla 653, Santiago 7800024, Chile; \\ areche@uchile.cl \\ 3 Departamento de Biotecnología, Universidad Autónoma Metropolitana-Iztapalapa. Av. San Rafael \\ Atlixco 186, Col. Vicentina C.P., 09340 Ciudad de México, Mexico \\ * Correspondence: cuhp@xanum.uam.mx; Tel.: +52-555-804-4600 (ext. 2846)
}

Academic Editor: Brendan M Duggan

Received: 25 January 2019; Accepted: 14 February 2019; Published: 15 February 2019

\begin{abstract}
Chromatographic separation combined with mass spectrometry is a powerful tool for the characterization of plant metabolites because of its high sensitivity and selectivity. In this work, the phytochemical profile of aerial and radicular parts of Coryphantha macromeris (Engelm.) Britton \& Rose growing under greenhouse conditions was qualitatively investigated for the first time by means of modern ultra-high-performance liquid chromatography-tandem mass spectrometry (UHPLC-PDA-HESI-Orbitrap-MS/MS). The UHPLC-PDA-HESI-Orbitrap-MS/MS analysis indicated a high complexity in phenolic metabolites. In our investigation, 69 compounds were detected and 60 of them were identified. Among detected compounds, several phenolic acids, phenolic glycosides, and organic acids were found. Within this diversity, 26 metabolites were exclusively detected in the aerial part, and 19 in the roots. Twenty-four metabolites occurred in both plant parts. According to the relative abundance of peaks in the chromatogram, ferulic and piscidic acids and their derivatives may correspond to one of the main phenolic compounds of $C$. macromeris. Our results contribute to the phytochemical knowledge regarding C. macromeris and its potential applications in the pharmaceutical and cosmetic industries. Besides, some metabolites and their fragmentation patterns are reported here for the first time for cacti species.
\end{abstract}

Keywords: Cactaceae; phenolic compounds; secondary metabolites; UHPLC; succulent plants; fragmentation pattern; active compounds

\section{Introduction}

The family Cactaceae is one of the most threatened within the plant kingdom [1], and is the most important plant family of the arid and semiarid regions of America [2], comprising around 1600 species [3]. Cacti are succulent plants which have been used for their functional properties and industrial utility [4-6]. Different cacti species obtained by cultivated or wild-collected methods have been used as food sources and fodder, for ornamental purposes, and for medicinal purposes (due to their antioxidant [7], antimutagenic [8], and bactericidal [9] properties), and have been cataloged as a source of bioactive compounds including alkaloids $[10,11]$ and phenolics $[12,13]$. 
Plants contain different classes of metabolites found in different concentrations and of a wide structural diversity. Their phytochemical profiles and functional properties may vary according to: a) the solvent and extraction processes [14,15], b) the stage of growth [16], c) storage conditions [17], and d) the specific section of the plant $[18,19]$. This complexity makes it difficult to identify plant metabolites through a single analytical method or under a defined separation system [20]. Several techniques have been proposed to achieve the elucidation of the plant metabolome, including the coupling of mass spectrometry with different separation techniques [21,22]. The knowledge of natural product chemistry is important since identified metabolites may serve as the basis for the discovery or design of novel compounds with biological activities [23] and as chemotaxonomic markers for plant classification [24].

The genus Coryphantha is highly endemic, distributed in the arid and semiarid regions located between the Sierra Madre Oriental and the Sierra Madre Occidental in northern Mexico, expanding to the southern regions of Arizona, Texas, and New Mexico, USA [25]. Currently, natural populations of Coryphantha species are reduced due to overexploitation and destruction of habitats. Coryphantha spp. are slow growing plants and the environmental conditions of semiarid regions make their germination, growth, and reproduction difficult; for this reason, biotechnological approaches have been proposed for the in vitro propagation of Coryphantha spp. and other cacti species [26,27]. Coryphantha macromeris (Engelm.) Britton \& Rose is a globose cactus traditionally known as "Dona Ana", "long mamma cory-cactus", "big needle cactus", and "biznaga partida-partida"; it is mainly distributed in northern Mexico and southern United States. In Mexico, C. macromeris is used in folk medicine for healing stomach disorders; nevertheless, hallucinogenic properties have been proposed, due to the presence of alkaloids such as macromerine [28] and other $\beta$-phenethylamine derivatives [29]. More recently, Kikuchi, et al. [30] evaluated the chemical constituents of a psychotropic herbal product and, using DNA sequence analysis, suggested that $C$. macromeris could be one of its components, as the rpl16 intron sequence of the herbal product showed $99 \%$ of similarity with that of C. macromeris. Information regarding the phytochemical composition and bioactive compounds in C. macromeris is scarce. Thus, the aim of this study was to identify, using UHPLC coupled with tandem mass spectrometry, the main secondary metabolites present in shoots and roots of Coryphantha macromeris growing under greenhouse conditions in order to contribute to the phytochemical knowledge of this species and its potential applications.

\section{Results and Discussion}

Methanolic extracts prepared with aerial and radicular parts of $C$. macromeris cultivated under greenhouse conditions were analyzed by ultra-high-performance liquid chromatography-tandem mass spectrometry and a photodiode array detector (UHPLC-PDA-HESI-Orbitrap-MS/MS). This technique has been used for the characterization of metabolites in complex mixtures [31], and the chromatographic conditions used in this work have shown efficiency for the separation and identification of different secondary metabolites [32-34]. For C. macromeris extracts, UHPLC conditions allowed a good separation of many sample components.

The phytochemical characterization of $C$. macromeris was achieved by comparing the obtained information by UHPLC-PDA-HESI-Orbitrap-MS/MS with spectroscopic evidences existing in the literature or by structure searching and studying the fragmentation pattern of molecules. Additionally, retention time and UV $\left(\lambda_{\max }\right)$ spectra were used for peak characterization. As far as we know, metabolite profiling of $C$. macromeris is reported for the first time in this work. Elution profile (Figure 1) and mass spectra obtained in negative ion mode indicated a highly complex phenolic composition. Peak characteristics (i.e., retention time, theoretical and measured mass, UV $\left(\lambda_{\max }\right)$, and fragmentation pattern) and tentative identification of each compound are summarized in Table 1. All detected compounds exhibited an accuracy smaller than $5 \mathrm{ppm}$. Under the proposed UHPLC-PDA-HESI-Orbitrap-MS/MS method, 69 compounds were separated, and 60 of them were tentatively identified in C. macromeris greenhouse plants (Table 1). Within this complexity, 26 compounds occurred in the aerial part, 19 in occurred in the roots, and 24 occurred in both plant parts, suggesting a translocation mechanism from actively photosynthetic tissues to metabolite consuming/storage areas. 


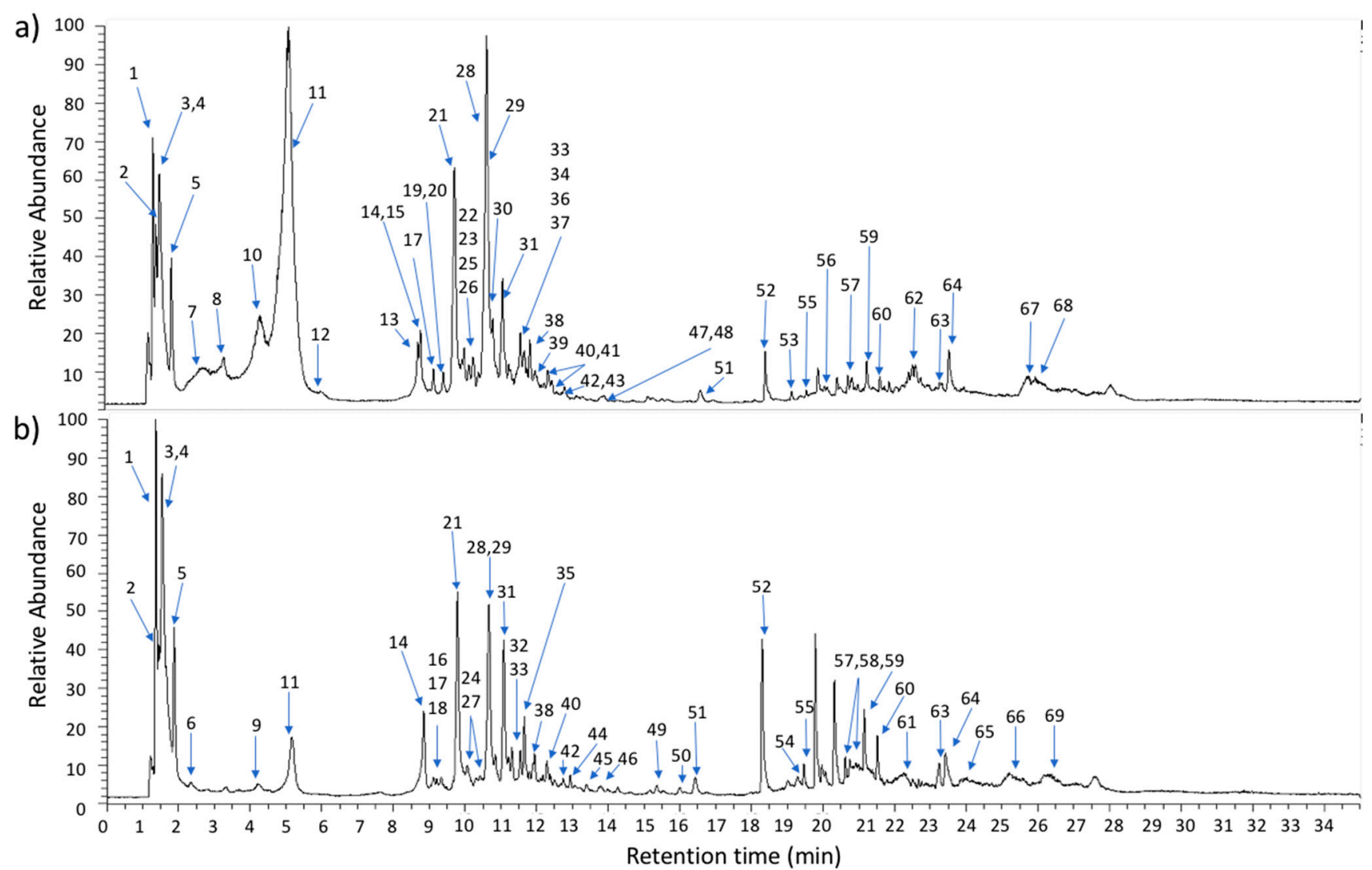

Figure 1. Ultra-high-performance liquid chromatography (UHPLC) chromatogram of Coryphantha macromeris methanolic extracts prepared with aerial (a) and radicular (b) parts. Peak numbers refer to the metabolites indicated in Table 1; repeated numbers in (a) or (b) indicate that the metabolite is found in both plant parts. 
Table 1. Metabolites identified in aerial and radicular parts of Coryphantha macromeris by ultra-high-performance liquid chromatography-tandem mass spectrometry (UHPLC-PDA-HESI-Orbitrap-MS/MS) data using heated electrospray ionization source (HESI) in negative ion mode.

\begin{tabular}{|c|c|c|c|c|c|c|c|c|c|}
\hline Peak & $\begin{array}{l}\text { Retention } \\
\text { Time (min) }\end{array}$ & $\begin{array}{l}\text { UVMax } \\
\left(\lambda_{\max }\right)\end{array}$ & Tentative Identification & $\begin{array}{c}\text { Elemental } \\
\text { Composition } \\
{[\mathrm{M}-\mathrm{H}]^{-}}\end{array}$ & $\begin{array}{l}\text { Theoretical } \\
\text { Mass }(m / z)\end{array}$ & $\begin{array}{c}\text { Measured } \\
\text { Mass } \\
(\mathrm{m} / \mathrm{z})\end{array}$ & $\begin{array}{l}\text { Accuracy } \\
\text { (ppm) }\end{array}$ & MSn Ions & Plant Part \\
\hline 1 & 1.37 & 220,272 & Vaccihein A & $\mathrm{C}_{18} \mathrm{H}_{17} \mathrm{O}_{9}^{-}$ & 377.08781 & 377.08600 & 4.80 & $\begin{array}{c}361.09076\left([\mathrm{M}-\mathrm{H}-\mathrm{OH}]^{-}\right) \\
347.07724\left(\left[\mathrm{M}-\mathrm{H}-\mathrm{OCH}_{3}\right]^{-}\right) \\
319.07990\left(\left[\mathrm{M}-\mathrm{H}-\mathrm{C}_{2} \mathrm{H}_{3} \mathrm{O}_{2}\right]^{-}\right) \\
289.06958\left(\left[\mathrm{M}-\mathrm{H}-\mathrm{C}_{2} \mathrm{H}_{3} \mathrm{O}_{2}-\mathrm{OCH}_{3}\right]^{-}\right) \\
125.02375\left(\left[\mathrm{M}-\mathrm{H}-\mathrm{C}_{8} \mathrm{H}_{9} \mathrm{O}_{4}-\mathrm{C}_{3} \mathrm{H}_{5} \mathrm{O}_{2}\right]^{-}\right)\end{array}$ & Root/shoot \\
\hline 2 & 1.45 & 220,272 & Dihydroxy methoxy butanoic acid & $\mathrm{C}_{5} \mathrm{H}_{9} \mathrm{O}_{5}^{-}$ & 149.04555 & 149.04498 & 3.82 & $\begin{array}{c}135.02925\left(\left[\mathrm{M}-\mathrm{H}-\mathrm{CH}_{3}\right]^{-}\right) \\
131.03433\left([\mathrm{M}-\mathrm{H}-\mathrm{OH}]^{-}\right) \\
119.03425\left(\left[\mathrm{M}-\mathrm{H}-\mathrm{CH}_{3}-\mathrm{OH}\right]^{-}\right) \\
103.03932\left(\left[\mathrm{M}-\mathrm{H}-\mathrm{OCH}_{3}-\mathrm{OH}\right]^{-}\right)\end{array}$ & Root/shoot \\
\hline 3 & 1.52 & 220,274 & 2-Hydroxy-succinic acid (malic acid) & $\mathrm{C}_{4} \mathrm{H}_{5} \mathrm{O}_{5}^{-}$ & 133.01390 & 133.01363 & 2.03 & $115.00297\left([\mathrm{M}-\mathrm{H}-\mathrm{OH}]^{-}\right)$ & Root/shoot \\
\hline 4 & 1.55 & 224,277 & Iso citric acid & $\mathrm{C}_{6} \mathrm{H}_{7} \mathrm{O}_{7}^{-}$ & 191.01973 & 191.01938 & 1.83 & $111.00790\left(\left[\mathrm{M}-\mathrm{H}-\mathrm{CO}_{2}-2 \mathrm{OH}\right]^{-}\right)$ & Root/shoot \\
\hline 5 & 1.87 & 277 & Iso citric acid isomer & $\mathrm{C}_{6} \mathrm{H}_{7} \mathrm{O}_{7}^{-}$ & 191.01973 & 191.01938 & 1.83 & $111.00790\left(\left[\mathrm{M}-\mathrm{H}-\mathrm{CO}_{2}-2 \mathrm{OH}\right]^{-}\right)$ & Root/shoot \\
\hline 6 & 2.35 & 220,277 & $\begin{array}{l}\text { 3,5-Dihydroxy-4-methyloxolan-2-yl } \\
\text { methoxy-6-hydroxymethyl oxane-3,4,5-triol }\end{array}$ & $\mathrm{C}_{12} \mathrm{H}_{21} \mathrm{O}_{9}^{-}$ & 309.11911 & 309.11935 & 0.78 & $\begin{array}{c}293.12424\left([\mathrm{M}-\mathrm{H}-\mathrm{OH}]^{-}\right) \\
279.10870\left(\left[\mathrm{M}-\mathrm{H}-\mathrm{OH}-\mathrm{CH}_{3}\right]^{-}\right) \\
147.06578\left(\left[\mathrm{M}-\mathrm{H}^{-} \mathrm{C}_{6} \mathrm{H}_{11} \mathrm{O}_{5}\right]^{-}\right) \\
131.07106\left(\left[\mathrm{M}-\mathrm{H}-\mathrm{C}_{6} \mathrm{H}_{11} \mathrm{O}_{5}-\mathrm{OH}\right]^{-}\right)\end{array}$ & Root \\
\hline 7 & 2.74 & 224,277 & Piscidic acid isomer & $\mathrm{C}_{11} \mathrm{H}_{11} \mathrm{O}_{7}^{-}$ & 255.05103 & 255.05098 & 0.20 & $\begin{array}{c}193.05013\left(\left[\mathrm{M}-\mathrm{H}-\mathrm{CHO}_{2}-\mathrm{OH}\right]^{-}\right) \\
165.05521\left(\left[\mathrm{M}-\mathrm{H}-\mathrm{C}_{2} \mathrm{H}_{2} \mathrm{O}_{3}-\mathrm{OH}\right]^{-}\right) \\
135.04442\left(\left[\mathrm{M}-\mathrm{H}-\mathrm{C}_{2} \mathrm{H}_{2} \mathrm{O}_{3}-\mathrm{CHO}_{2}\right]^{-}\right) \\
119.04952\left(\left[\mathrm{M}-\mathrm{H}-\mathrm{C}_{2} \mathrm{H}_{2} \mathrm{O}_{3}-\mathrm{CH}_{2}-\mathrm{OH}\right]^{-}\right) \\
107.04927\left(\left[\mathrm{M}-\mathrm{H}-\mathrm{C}_{4} \mathrm{H}_{4} \mathrm{O}_{3}\right]^{-}\right)\end{array}$ & Shoot \\
\hline 8 & 3.34 & 228,277 & Piscidic acid isomer & $\mathrm{C}_{11} \mathrm{H}_{11} \mathrm{O}_{7}^{-}$ & 255.05103 & 255.05098 & 0.20 & $\begin{array}{c}193.05013\left(\left[\mathrm{M}-\mathrm{H}-\mathrm{CHO}_{2}-\mathrm{OH}\right]^{-}\right) \\
165.05521\left(\left[\mathrm{M}-\mathrm{H}-\mathrm{C}_{2} \mathrm{H}_{2} \mathrm{O}_{3}-\mathrm{OH}\right]^{-}\right) \\
\left.135.04442\left(\left[\mathrm{M}-\mathrm{H}-\mathrm{C}_{2} \mathrm{H}_{2} \mathrm{O}_{3}-\mathrm{CHO}\right]_{2}\right]^{-}\right) \\
119.04952\left(\left[\mathrm{M}-\mathrm{H}-\mathrm{C}_{2} \mathrm{H}_{2} \mathrm{O}_{3}-\mathrm{CH}_{2}-\mathrm{OH}\right]^{-}\right) \\
107.04927\left(\left[\mathrm{M}-\mathrm{H}-\mathrm{C}_{4} \mathrm{H}_{4} \mathrm{O}_{3}\right]^{-}\right)\end{array}$ & Shoot \\
\hline 9 & 4.22 & $\begin{array}{l}197,223 \\
278\end{array}$ & Protocatechuic acid hesoxide & $\mathrm{C}_{13} \mathrm{H}_{15} \mathrm{O}_{9}^{-}$ & 315.07230 & 315.07239 & 0.29 & $\begin{array}{c}255.05104\left(\left[\mathrm{M}-\mathrm{H}-\mathrm{C}_{2} \mathrm{H}_{5} \mathrm{O}_{2}\right]^{-}\right) \\
211.06094\left(\left[\mathrm{M}-\mathrm{H}-\mathrm{C}_{2} \mathrm{H}_{5} \mathrm{O}_{2}-\mathrm{CHO}_{2}\right]^{-}\right) \\
153.01878\left(\left[\mathrm{M}-\mathrm{H}^{-} \mathrm{C}_{6} \mathrm{H}_{11} \mathrm{O}_{5}\right]^{-}\right) \\
137.02385\left(\left[\mathrm{M}-\mathrm{H}-\mathrm{C}_{6} \mathrm{H}_{11} \mathrm{O}_{5}-\mathrm{OH}\right]^{-}\right) \\
121.02895\left(\left[\mathrm{M}-\mathrm{H}-\mathrm{C}_{6} \mathrm{H}_{11} \mathrm{O}_{5}-2 \mathrm{OH}\right]^{-}\right) \\
109.02863\left(\left[\mathrm{M}-\mathrm{H}-\mathrm{C}_{6} \mathrm{H}_{11} \mathrm{O}_{5}-\mathrm{CHO}_{2}\right]^{-}\right)\end{array}$ & Root \\
\hline 10 & 4.34 & 223,276 & Piscidic acid isomer & $\mathrm{C}_{11} \mathrm{H}_{11} \mathrm{O}_{7}^{-}$ & 255.05103 & 255.05098 & 0.20 & $\begin{array}{c}193.05013\left(\left[\mathrm{M}-\mathrm{H}-\mathrm{CHO}_{2}-\mathrm{OH}\right]^{-}\right) \\
165.05521\left(\left[\mathrm{M}-\mathrm{H}-\mathrm{C}_{2} \mathrm{H}_{2} \mathrm{O}_{3}-\mathrm{OH}\right]^{-}\right)\end{array}$ & Shoot \\
\hline
\end{tabular}


Table 1. Cont

\begin{tabular}{|c|c|c|c|c|c|c|c|c|c|}
\hline Peak & $\begin{array}{l}\text { Retention } \\
\text { Time (min) }\end{array}$ & $\begin{array}{l}\text { UVMax } \\
\left(\lambda_{\max }\right)\end{array}$ & Tentative Identification & $\begin{array}{c}\text { Elemental } \\
\text { Composition } \\
{[\mathrm{M}-\mathrm{H}]^{-}}\end{array}$ & $\begin{array}{l}\text { Theoretical } \\
\text { Mass }(m / z)\end{array}$ & $\begin{array}{l}\text { Measured } \\
\text { Mass } \\
(\mathrm{m} / \mathrm{z})\end{array}$ & $\begin{array}{l}\text { Accuracy } \\
\text { (ppm) }\end{array}$ & MSn Ions & Plant Part \\
\hline & & & & & & & & $\begin{array}{c}135.04442\left(\left[\mathrm{M}-\mathrm{H}-\mathrm{C}_{2} \mathrm{H}_{2} \mathrm{O}_{3}-\mathrm{CHO}_{2}\right]^{-}\right) \\
119.04952\left(\left[\mathrm{M}-\mathrm{H}-\mathrm{C}_{2} \mathrm{H}_{2} \mathrm{O}_{3}-\mathrm{CH}_{2}-\mathrm{OH}\right]^{-}\right) \\
107.04927\left(\left[\mathrm{M}-\mathrm{H}-\mathrm{C}_{4} \mathrm{H}_{4} \mathrm{O}_{3}\right]^{-}\right)\end{array}$ & \\
\hline 11 & 5.13 & 222,275 & Piscidic acid isomer & $\mathrm{C}_{11} \mathrm{H}_{11} \mathrm{O}_{7}^{-}$ & 255.05103 & 255.05095 & 0.31 & $\begin{array}{c}193.05013\left(\left[\mathrm{M}-\mathrm{H}-\mathrm{CHO}_{2}-\mathrm{OH}\right]^{-}\right) \\
165.05521\left(\left[\mathrm{M}-\mathrm{H}-\mathrm{C}_{2} \mathrm{H}_{2} \mathrm{O}_{3}-\mathrm{OH}\right]^{-}\right) \\
135.04442\left(\left[\mathrm{M}-\mathrm{H}-\mathrm{C}_{2} \mathrm{H}_{2} \mathrm{O}_{3}-\mathrm{CHO}_{2}\right]^{-}\right) \\
119.04952\left(\left[\mathrm{M}-\mathrm{H}-\mathrm{C}_{2} \mathrm{H}_{2} \mathrm{O}_{3}-\mathrm{CH}_{2}-\mathrm{OH}\right]^{-}\right) \\
107.04927\left(\left[\mathrm{M}-\mathrm{H}-\mathrm{C}_{4} \mathrm{H}_{4} \mathrm{O}_{3}\right]^{-}\right)\end{array}$ & Root/shoot \\
\hline 12 & 6.02 & 222,275 & Piscidic acid isomer & $\mathrm{C}_{11} \mathrm{H}_{11} \mathrm{O}_{7}^{-}$ & 255.05103 & 255.05101 & 0.08 & $\begin{array}{c}193.05013\left(\left[\mathrm{M}-\mathrm{H}-\mathrm{CHO}_{2}-\mathrm{OH}\right]^{-}\right) \\
165.05521\left(\left[\mathrm{M}-\mathrm{H}-\mathrm{C}_{2} \mathrm{H}_{2} \mathrm{O}_{3}-\mathrm{OH}\right]^{-}\right) \\
135.04442\left(\left[\mathrm{M}-\mathrm{H}-\mathrm{C}_{2} \mathrm{H}_{2} \mathrm{O}_{3}-\mathrm{CHO}_{2}\right]^{-}\right) \\
119.04952\left(\left[\mathrm{M}-\mathrm{H}-\mathrm{C}_{2} \mathrm{H}_{2} \mathrm{O}_{3}-\mathrm{CH}_{2}-\mathrm{OH}\right]^{-}\right) \\
107.04927\left(\left[\mathrm{M}-\mathrm{H}-\mathrm{C}_{4} \mathrm{H}_{4} \mathrm{O}_{3}\right]^{-}\right)\end{array}$ & Shoot \\
\hline 13 & 8.74 & 222,277 & Lucuminic acid & $\mathrm{C}_{19} \mathrm{H}_{25} \mathrm{O}_{12}^{-}$ & 445.13515 & 445.13531 & 0.36 & $\begin{array}{c}163.03947\left(\left[\mathrm{M}-\mathrm{H}-\mathrm{C}_{10} \mathrm{H}_{19} \mathrm{O}_{8}-\mathrm{OH}\right]^{-}\right) \\
119.04939\left(\left[\mathrm{M}-\mathrm{H}-\mathrm{C}_{11} \mathrm{H}_{19} \mathrm{O}_{10}-\mathrm{OH}\right]^{-}\right) \\
107.04942\left(\left[\mathrm{M}-\mathrm{H}-\mathrm{C}_{11} \mathrm{H}_{19} \mathrm{O}_{9}-\mathrm{CHO}_{2}\right]^{-}\right)\end{array}$ & Shoot \\
\hline 14 & 8.85 & 223,276 & Hyrtioerectine C & $\mathrm{C}_{11} \mathrm{H}_{12} \mathrm{NO}_{4}^{-}$ & 222.07718 & 222.07703 & 0.68 & $\begin{array}{c}206.08206\left([\mathrm{M}-\mathrm{H}-\mathrm{OH}]^{-}\right) \\
198.07718\left(\left[\mathrm{M}-\mathrm{H}-\mathrm{C}_{2} \mathrm{H}_{3}\right]^{-}\right) \\
180.06580\left(\left[\mathrm{M}-\mathrm{H}-\mathrm{C}_{2} \mathrm{H}_{3}-\mathrm{OH}\right]^{-}\right) \\
178.08685\left(\left[\mathrm{M}-\mathrm{H}-\mathrm{CHO}_{2}\right]^{-}\right)\end{array}$ & Root/shoot \\
\hline 15 & 8.93 & 227,283 & Piscidic acid isomer & $\mathrm{C}_{11} \mathrm{H}_{11} \mathrm{O}_{7}^{-}$ & 255.05103 & 255.05104 & 0.04 & $\begin{array}{c}193.05013\left(\left[\mathrm{M}-\mathrm{H}-\mathrm{CHO}_{2}-\mathrm{OH}\right]^{-}\right) \\
165.05521\left(\left[\mathrm{M}-\mathrm{H}-\mathrm{C}_{2} \mathrm{H}_{2} \mathrm{O}_{3}-\mathrm{OH}\right]^{-}\right) \\
135.04442\left(\left[\mathrm{M}-\mathrm{H}-\mathrm{C}_{2} \mathrm{H}_{2} \mathrm{O}_{3}-\mathrm{CHO}_{2}\right]^{-}\right) \\
119.04952\left(\left[\mathrm{M}-\mathrm{H}-\mathrm{C}_{2} \mathrm{H}_{2} \mathrm{O}_{3}-\mathrm{CH}_{2}-\mathrm{OH}\right]^{-}\right) \\
107.04927\left(\left[\mathrm{M}-\mathrm{H}-\mathrm{C}_{4} \mathrm{H}_{4} \mathrm{O}_{3}\right]^{-}\right)\end{array}$ & Shoot \\
\hline 16 & 9.12 & 255,207 & Protocatechuic aldehyde & $\mathrm{C}_{7} \mathrm{H}_{5} \mathrm{O}_{3}{ }^{-}$ & 137.02442 & 137.02386 & 4.09 & $\begin{array}{c}121.02882\left([\mathrm{M}-\mathrm{H}-\mathrm{OH}]^{-}\right) \\
109.02884\left([\mathrm{M}-\mathrm{H}-\mathrm{COH}]^{-}\right)\end{array}$ & Root \\
\hline 17 & 9.19 & 230,286 & Piscidic acid derivative & $\mathrm{C}_{21} \mathrm{H}_{27} \mathrm{O}_{13}^{-}$ & - & 487.14600 & - & $\begin{array}{c}255.05110 \text { ([piscidic acid] }^{-} \text {) } \\
\left.193.05078 \text { ([piscidic acid-CHO } \mathrm{CH}_{2}-\mathrm{OH}\right]^{-} \text {) } \\
\left.165.05516 \text { ([piscidic acid- } \mathrm{C}_{2} \mathrm{H}_{2} \mathrm{O}_{3}-\mathrm{OH}\right]^{-} \\
\left.135.04453 \text { ([piscidic acid- } \mathrm{C}_{2} \mathrm{H}_{2} \mathrm{O}_{3}-\mathrm{CHO}_{2}\right]^{-} \text {) } \\
\left.107.04935 \text { ([piscidic acid- } \mathrm{C}_{4} \mathrm{H}_{4} \mathrm{O}_{3}\right]^{-} \text {) }\end{array}$ & Root/shoot \\
\hline 18 & 9.41 & 223,278 & Piscidic acid derivative & $\mathrm{C}_{20} \mathrm{H}_{27} \mathrm{O}_{13}^{-}$ & - & 475.14606 & - & $\begin{array}{c}\text { 255.05112 ([piscidic acid }]^{-} \text {) } \\
\left.\left.\text { 193.05037 ([piscidic acid- } \mathrm{CHO}_{2}-\mathrm{OH}\right]^{-}\right) \\
\left.\left.\text {165.05513([piscidic acid- } \mathrm{C}_{2} \mathrm{H}_{2} \mathrm{O}_{3}-\mathrm{OH}\right]^{-}\right) \\
\left.\text {135.04453([piscidic acid- } \mathrm{C}_{2} \mathrm{H}_{2} \mathrm{O}_{3}-\mathrm{CHO}_{2}\right]^{-} \text {) } \\
\left.\left.\text { 107.04923 ([piscidic acid- } \mathrm{C}_{4} \mathrm{H}_{4} \mathrm{O}_{3}\right]^{-}\right)\end{array}$ & Root \\
\hline
\end{tabular}


Table 1. Cont.

\begin{tabular}{|c|c|c|c|c|c|c|c|c|c|}
\hline Peak & $\begin{array}{l}\text { Retention } \\
\text { Time (min) }\end{array}$ & $\begin{array}{l}\text { UVMax } \\
\left(\lambda_{\max }\right)\end{array}$ & Tentative Identification & $\begin{array}{l}\text { Elemental } \\
\text { Composition } \\
{[\mathrm{M}-\mathrm{H}]^{-}}\end{array}$ & $\begin{array}{l}\text { Theoretical } \\
\text { Mass }(m / z)\end{array}$ & $\begin{array}{l}\text { Measured } \\
\text { Mass } \\
(\mathrm{m} / \mathrm{z})\end{array}$ & $\begin{array}{l}\text { Accuracy } \\
\text { (ppm) }\end{array}$ & MSn Ions & Plant Part \\
\hline 19 & 9.45 & 231,295 & Sinapic acid derivative & $\mathrm{C}_{22} \mathrm{H}_{29} \mathrm{O}_{14}^{-}$ & - & 517.15649 & - & $\begin{array}{c}223.06104\left([\text { sinapic acid }]^{-}\right) \\
208.03767\left(\left[\text { sinapic acid- } \mathrm{CH}_{3}\right]^{-}\right) \\
179.07083\left(\left[\text { sinapic acid- } \mathrm{CHO}_{2}\right]^{-}\right) \\
164.04738\left(\left[\text { sinapic acid- } \mathrm{CHO}_{2}-\mathrm{OH}\right]^{-}\right)\end{array}$ & Shoot \\
\hline 20 & 9.50 & 231,295 & Sinapic acid & $\mathrm{C}_{11} \mathrm{H}_{11} \mathrm{O}_{5}^{-}$ & 223.06070 & 223.06102 & 1.43 & $\begin{array}{c}208.03757\left(\left[\mathrm{M}-2 \mathrm{H}-\mathrm{CH}_{3}\right]^{-}\right) \\
179.07094\left(\left[\mathrm{M}-\mathrm{H}^{-} \mathrm{CHO}_{2}\right]^{-}\right) \\
164.04730\left(\left[\mathrm{M}-2 \mathrm{H}-\mathrm{CHO}_{2}-\mathrm{OH}\right]^{-}\right)\end{array}$ & Shoot \\
\hline 21 & 9.77 & 224,276 & Syringic acid acetate & $\mathrm{C}_{11} \mathrm{H}_{11} \mathrm{O}_{6}^{-}$ & 239.05611 & 239.05594 & 0.71 & $\begin{array}{c}197.04517\left([\text { syringic acid }]^{-}\right) \\
195.06580\left(\left[\mathrm{M}-\mathrm{H}-\mathrm{CHO}_{2}\right]^{-}\right) \\
179.03439\left(\left[\mathrm{M}-\mathrm{H}-2 \mathrm{CH}_{3} \mathrm{O}\right]^{-}\right) \\
\left.149.06023\left[\mathrm{M}-\mathrm{H}-2 \mathrm{CH}_{3} \mathrm{O}-\mathrm{OH}\right]^{-}\right) \\
135.04456\left(\left[\mathrm{M}-\mathrm{H}-\mathrm{CHO}_{2}-2 \mathrm{CH}_{3} \mathrm{O}\right]^{-}\right) \\
107.04944\left(\left[\mathrm{M}-\mathrm{H}-\mathrm{CHO}_{2}-\mathrm{CH}_{3} \mathrm{O}-\mathrm{C}_{2} \mathrm{H}_{3} \mathrm{O}_{2}\right]^{-}\right)\end{array}$ & Root/shoot \\
\hline 22 & 9.97 & 231,286 & Piscidic acid derivative & $\mathrm{C}_{21} \mathrm{H}_{27} \mathrm{O}_{13}^{-}$ & - & 487.14603 & - & $\begin{array}{c}255.05101 \text { ([piscidic acid] }{ }^{-} \text {) } \\
\left.193.05025\left(\text { [piscidic acid- } \mathrm{CHO}_{2}-\mathrm{OH}\right]^{-}\right) \\
\left.165.05528\left(\text { [piscidic acid- } \mathrm{C}_{2} \mathrm{H}_{2} \mathrm{O}_{3}-\mathrm{OH}\right]^{-}\right) \\
135.04456\left(\text { [piscidic acid- } \mathrm{C}_{2} \mathrm{H}_{2} \mathrm{O}_{3}-\mathrm{CHO}_{2}\right]^{-} \text {) } \\
107.04945\left(\left[\text { piscidic acid- } \mathrm{C}_{4} \mathrm{H}_{4} \mathrm{O}_{3}\right]^{-}\right)\end{array}$ & Shoot \\
\hline 23 & 10.04 & 233,283 & Syringic acid acetate derivative & $\mathrm{C}_{19} \mathrm{H}_{31} \mathrm{O}_{8}^{-}$ & - & 387.20276 & - & 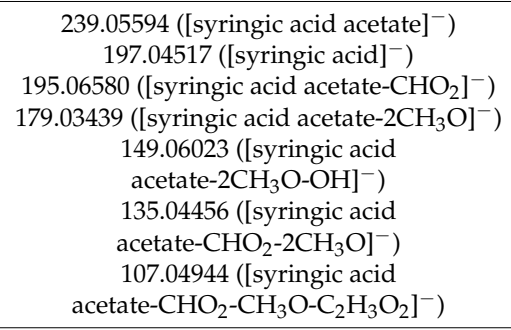 & Shoot \\
\hline 24 & 10.08 & 231,283 & Caffeic acid & $\mathrm{C}_{9} \mathrm{H}_{7} \mathrm{O}_{4}^{-}$ & 179.03498 & 179.03477 & 1.17 & $\begin{array}{c}163.03950\left([\mathrm{M}-\mathrm{H}-\mathrm{OH}]^{-}\right) \\
135.04510\left(\left[\mathrm{M}-\mathrm{H}-\mathrm{CHO}_{2}\right]^{-}\right) \\
109.02870\left(\left[\mathrm{M}-\mathrm{H}-\mathrm{C}_{3} \mathrm{H}_{3} \mathrm{O}_{2}\right]^{-}\right)\end{array}$ & Root \\
\hline 25 & 10.17 & 231,286 & Sinapic acid hexoside & $\mathrm{C}_{17} \mathrm{H}_{21} \mathrm{O}_{10}-$ & 385.11402 & 385.11438 & 0.93 & $\begin{array}{c}223.06099\left(\left[\mathrm{M}-\mathrm{H}-\mathrm{C}_{6} \mathrm{H}_{11} \mathrm{O}_{5}\right]^{-}\right) \\
208.03757\left(\left[\text { sinapic acid- } \mathrm{CH}_{3}\right]^{-}\right) \\
179.07095\left(\left[\text { sinapic acid- } \mathrm{CHO}_{2}\right]^{-}\right) \\
164.04745\left(\left[\text { sinapic acid- } \mathrm{CHO}_{2}-\mathrm{OH}\right]^{-}\right)\end{array}$ & Shoot \\
\hline 26 & 10.27 & 234,283 & $\begin{array}{c}\text { Propanedioic acid, [5-[[2-[(6-deoxy- } \\
\alpha \text {-L-galactopyranosyl) oxy] cyclohexyl] } \\
\text { oxy]-Pentyl] }\end{array}$ & $\mathrm{C}_{20} \mathrm{H}_{33} \mathrm{O}_{10}^{-}$ & 433.20792 & 433.20825 & 0.76 & $\begin{array}{c}417.21347\left([\mathrm{M}-\mathrm{H}-\mathrm{OH}]^{-}\right) \\
387.20309\left(\left[\mathrm{M}-\mathrm{H}-\mathrm{CHO}_{2}\right]^{-}\right)\end{array}$ & Shoot \\
\hline
\end{tabular}


Table 1. Cont

\begin{tabular}{|c|c|c|c|c|c|c|c|c|c|}
\hline Peak & $\begin{array}{l}\text { Retention } \\
\text { Time (min) }\end{array}$ & $\begin{array}{l}\text { UVMax } \\
\left(\lambda_{\max }\right)\end{array}$ & Tentative Identification & $\begin{array}{c}\text { Elemental } \\
\text { Composition } \\
{[\mathrm{M}-\mathrm{H}]^{-}}\end{array}$ & $\begin{array}{l}\text { Theoretical } \\
\text { Mass }(\mathrm{m} / \mathrm{z})\end{array}$ & $\begin{array}{l}\text { Measured } \\
\text { Mass } \\
(\mathrm{m} / \mathrm{z}) \\
\end{array}$ & $\begin{array}{l}\text { Accuracy } \\
\text { (ppm) }\end{array}$ & MSn Ions & Plant Part \\
\hline & & & & & & & & $\begin{array}{l}287.15030\left(\left[\mathrm{M}-\mathrm{H}-\mathrm{C}_{6} \mathrm{H}_{11} \mathrm{O}_{4}\right]^{-}\right) \\
245.13950\left(\left[\mathrm{M}-\mathrm{H}-\mathrm{C}_{8} \mathrm{H}_{13} \mathrm{O}_{5}\right]^{-}\right) \\
131.07069\left(\left[\mathrm{M}-\mathrm{H}-\mathrm{C}_{14} \mathrm{H}_{23} \mathrm{O}_{6}\right]^{-}\right)\end{array}$ & \\
\hline 27 & 10.31 & 237,283 & $\begin{array}{l}\text { Cyclohexanecarboxylic acid, } \\
\text { 3-[(6-deoxy-3-O-methyl-D-galactopyranosyl)oxy }\end{array}$ & 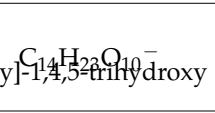 & 351.12967 & 351.13010 & 1.22 & $\begin{array}{c}303.14487\left([\mathrm{M}-\mathrm{H}-3 \mathrm{OH}]^{-}\right) \\
287.14999\left([\mathrm{M}-\mathrm{H}-4 \mathrm{OH}]^{-}\right) \\
273.13449\left(\left[\mathrm{M}-\mathrm{H}-3 \mathrm{OH}-\mathrm{CH}_{3} \mathrm{O}\right]^{-}\right)\end{array}$ & Root \\
\hline 28 & 10.63 & 235,326 & Ferulic acid & $\mathrm{C}_{10} \mathrm{H}_{9} \mathrm{O}_{4}^{-}$ & 193.05063 & 193.05032 & 1.61 & $\begin{array}{c}179.03455\left(\left[\mathrm{M}-\mathrm{H}_{-}-\mathrm{CH}_{3}\right]^{-}\right) \\
149.06050\left(\left[\mathrm{M}-\mathrm{H}-\mathrm{CHO}_{2}\right]^{-}\right) \\
163.03963\left(\left[\mathrm{M}-\mathrm{H}-\mathrm{CH}_{3}-\mathrm{OH}\right]^{-}\right) \\
147.04456\left(\left[\mathrm{M}-\mathrm{H}-\mathrm{CH}_{3}-2 \mathrm{OH}\right]^{-}\right)\end{array}$ & Root/shoot \\
\hline 29 & 10.69 & 235,327 & Ferulic acid derivative (fertaric acid) & $\mathrm{C}_{14} \mathrm{H}_{13} \mathrm{O}_{9}{ }^{-}$ & 325.05651 & 325.05664 & 0.40 & $\begin{array}{c}193.05032\left(\left[\text { ferulic acid] }{ }^{-} \text {) }\right.\right. \\
179.03453\left(\left[\text { ferulic acid- }-\mathrm{CH}_{3}\right]^{-} \text {) }\right. \\
163.03954\left(\left[\text { ferulic acid- } \mathrm{CH}_{3}-\mathrm{OH}\right]^{-} \text {) }\right.\end{array}$ & Root/shoot \\
\hline 30 & 10.84 & 237,291 & $\begin{array}{l}\text { 7,8,11-Trihydroxyguai-4-en- 3-one } \\
\text { 8-O- } \beta \text {-D-glucopyranoside }\end{array}$ & $\mathrm{C}_{21} \mathrm{H}_{33} \mathrm{O}_{9}^{-}$ & 429.21250 & 429.21335 & 1.98 & $\begin{array}{c}267.16003\left(\left[\mathrm{M}-\mathrm{H}_{-} \mathrm{C}_{6} \mathrm{H}_{11} \mathrm{O}_{5}\right]^{-}\right) \\
249.14989\left(\left[\mathrm{M}-\mathrm{H}-\mathrm{C}_{6} \mathrm{H}_{11} \mathrm{O}_{5}-\mathrm{OH}\right]^{-}\right)\end{array}$ & Shoot \\
\hline 31 & 11.09 & 235,286 & Cinnamic acid derivative & $\mathrm{C}_{8} \mathrm{H}_{14} \mathrm{O}_{6}{ }^{-}$ & - & 206.08205 & - & $\begin{array}{c}147.04449\left([\text { cinnamic acid }]^{-}\right) \\
103.05447\left(\left[\text { cinnamic acid- }-\mathrm{CHO}_{2}\right]^{-}\right)\end{array}$ & Root/shoot \\
\hline 32 & 11.22 & 227,283 & $\begin{array}{c}\text { 2-Propenoic acid, 2-methyl-, } \\
\text { 4-[2-(2,4-dioxo-1,5-dioxaspiro } \\
\text { [5.5]undec-3-yl)ethenyl]-6-(2,4-dioxo-1,5-dioxasp } \\
\text { ester }\end{array}$ & 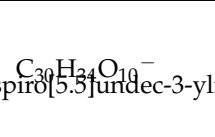 & lidene)-4-hexeny & $y^{554.21448}$ & 3.27 & $193.05049\left(\left[\mathrm{M}-\mathrm{H}-\mathrm{C}_{20} \mathrm{H}_{25} \mathrm{O}_{6}\right]^{-}\right)$ & Root \\
\hline 33 & 11.54 & $\begin{array}{l}239,289 \\
323\end{array}$ & Ferulic acid isomer & $\mathrm{C}_{10} \mathrm{H}_{9} \mathrm{O}_{4}^{-}$ & 193.05063 & 193.05048 & 0.78 & $\begin{array}{c}179.03458\left(\left[\mathrm{M}-\mathrm{H}-\mathrm{CH}_{3}\right]^{-}\right) \\
149.06030\left(\left[\mathrm{M}-\mathrm{H}-\mathrm{CHO}_{2}\right]^{-}\right) \\
163.03954\left(\left[\mathrm{M}-\mathrm{H}-\mathrm{CH}_{3}-\mathrm{OH}\right]^{-}\right)\end{array}$ & Root/shoot \\
\hline 34 & 11.61 & $\begin{array}{l}238,292 \\
323\end{array}$ & Ferulic acid isomer & $\mathrm{C}_{10} \mathrm{H}_{9} \mathrm{O}_{4}^{-}$ & 193.05063 & 193.05038 & 1.29 & $\begin{array}{c}179.03441\left(\left[\mathrm{M}-\mathrm{H}_{-}-\mathrm{CH}_{3}\right]^{-}\right) \\
149.06026\left(\left[\mathrm{M}-\mathrm{H}-\mathrm{CHO}_{2}\right]^{-}\right) \\
163.03937\left(\left[\mathrm{M}-\mathrm{H}-\mathrm{CH}_{3}-\mathrm{OH}\right]^{-}\right) \\
147.04457\left(\left[\mathrm{M}-\mathrm{H}-\mathrm{CH}_{3}-2 \mathrm{OH}\right]^{-}\right)\end{array}$ & Shoot \\
\hline 35 & 11.66 & 226,282 & Ferulic acid derivative I & $\mathrm{C}_{24} \mathrm{H}_{24} \mathrm{O}_{5}^{-}$ & - & 392.16193 & - & $\begin{array}{c}193.05038\left(\left[\text { ferulic acid] }{ }^{-}\right)\right. \\
149.06030\left(\left[\text { ferulic acid- } \mathrm{CHO}_{2}\right]^{-}\right) \\
163.03954\left(\left[\text { ferulic acid- } \mathrm{CH}_{3}-\mathrm{OH}\right]^{-}\right)\end{array}$ & Root \\
\hline 36 & 11.71 & 236,286 & Ferulic acid isomer & $\mathrm{C}_{10} \mathrm{H}_{9} \mathrm{O}_{4}^{-}$ & 193.05063 & 193.05038 & 1.29 & $\begin{array}{c}179.03452\left(\left[\mathrm{M}-\mathrm{H}-\mathrm{CH}_{3}\right]^{-}\right) \\
149.06024\left(\left[\mathrm{M}-\mathrm{H}-\mathrm{CHO}_{2}\right]^{-}\right) \\
163.03937\left(\left[\mathrm{M}-\mathrm{H}-\mathrm{CH}_{3}-\mathrm{OH}\right]^{-}\right) \\
147.04440\left(\left[\mathrm{M}-\mathrm{H}-\mathrm{CH}_{3}-2 \mathrm{OH}\right]^{-}\right) \\
\end{array}$ & Shoot \\
\hline
\end{tabular}


Table 1. Cont

\begin{tabular}{|c|c|c|c|c|c|c|c|c|c|}
\hline Peak & $\begin{array}{l}\text { Retention } \\
\text { Time (min) }\end{array}$ & $\begin{array}{l}\text { UVMax } \\
\left(\lambda_{\max }\right)\end{array}$ & Tentative Identification & $\begin{array}{c}\text { Elemental } \\
\text { Composition } \\
\text { [M-H }^{-}\end{array}$ & $\begin{array}{l}\text { Theoretical } \\
\text { Mass }(m / z)\end{array}$ & $\begin{array}{l}\text { Measured } \\
\text { Mass } \\
(\mathrm{m} / \mathrm{z})\end{array}$ & $\begin{array}{l}\text { Accuracy } \\
\text { (ppm) }\end{array}$ & MSn Ions & Plant Part \\
\hline 37 & 11.79 & $\begin{array}{l}235,286 \\
380\end{array}$ & 2-Isoferulic piscidic acid-1-metyl ester & $\mathrm{C}_{22} \mathrm{H}_{21} \mathrm{O}_{10}{ }^{-}$ & 445.11400 & 445.11438 & 0.85 & $\begin{array}{c}255.05092 \text { ([piscidic acid }]^{-} \text {) } \\
\left.193.050380 \text { ([ferulic acid] }]^{-}\right) \\
\left.\left.165.05516 \text { ([piscidic acid- } \mathrm{C}_{2} \mathrm{H}_{2} \mathrm{O}_{3}-\mathrm{OH}\right]^{-}\right) \\
135.04440\left(\text { [piscidic acid- } \mathrm{C}_{2} \mathrm{H}_{2} \mathrm{O}_{3}-\mathrm{CHO}_{2}\right]^{-} \text {) } \\
\left.107.04936 \text { ([piscidic acid- } \mathrm{C}_{4} \mathrm{H}_{4} \mathrm{O}_{3}\right]^{-} \text {) }\end{array}$ & Shoot \\
\hline 38 & 11.86 & $\begin{array}{l}240,296 \\
381\end{array}$ & Ferulic acid derivative II & $\mathrm{C}_{20} \mathrm{H}_{29} \mathrm{O}_{10}^{-}$ & - & 429.17707 & - & $\begin{array}{c}\left.193.05029 \text { ([ferulic acid }]^{-}\right) \\
179.03450\left(\left[\text { ferulic acid- } \mathrm{CH}_{3}\right]^{-}\right) \\
163.03937\left(\left[\text { ferulic acid- } \mathrm{CH}_{3}-\mathrm{OH}\right]^{-}\right) \\
\left.147.04440\left(\text { fferulic acid- } \mathrm{CH}_{3}-2 \mathrm{OH}\right]^{-}\right)\end{array}$ & Root/shoot \\
\hline 39 & 12.18 & 283,368 & Ferulic acid derivative III & $\mathrm{C}_{21} \mathrm{H}_{31} \mathrm{O}_{13}^{-}$ & - & 491.17731 & - & $\begin{array}{c}193.05026\left([\text { ferulic acid }]^{-}\right) \\
179.03456\left(\left[\text { ferulic acid- } \mathrm{CH}_{3}\right]^{-}\right) \\
163.03929\left(\left[\text { ferulic acid- } \mathrm{CH}_{3}-\mathrm{OH}\right]^{-}\right) \\
147.04446\left(\left[\text { ferulic acid- }-\mathrm{CH}_{3}-2 \mathrm{OH}\right]^{-}\right)\end{array}$ & Shoot \\
\hline 40 & 12.38 & 283 & Azelaic acid & $\mathrm{C}_{9} \mathrm{H}_{15} \mathrm{O}_{4}^{-}$ & 187.09758 & 187.09740 & 0.96 & $\begin{array}{c}169.08130\left([\mathrm{M}-\mathrm{H}-\mathrm{OH}]^{-}\right) \\
125.09650\left(\left[\mathrm{M}-\mathrm{H}-\mathrm{CHO}_{2}-\mathrm{OH}\right]^{-}\right)\end{array}$ & Root/shoot \\
\hline 41 & 12.47 & 284,368 & Ferulic acid derivative IV & $\mathrm{C}_{20} \mathrm{H}_{29} \mathrm{O}_{10}{ }^{-}$ & - & 429.17706 & - & $\begin{array}{c}193.05023\left([\text { ferulic acid }]^{-}\right) \\
179.03427\left(\left[\text { ferulic acid- }-\mathrm{CH}_{3}\right]^{-}\right) \\
163.03958\left(\left[\text { ferulic acid- } \mathrm{CH}_{3}-\mathrm{OH}\right]^{-} \text {) }\right. \\
147.04450\left(\left[\text { ferulic acid- } \mathrm{CH}_{3}-2 \mathrm{OH}\right]^{-} \text {) }\right.\end{array}$ & Shoot \\
\hline 42 & 12.70 & 259 & 2-Phenylethyl $\beta$-D-glucopyranoside & $\mathrm{C}_{14} \mathrm{H}_{19} \mathrm{O}_{6}{ }^{-}$ & 283.11871 & 283.11893 & 0.78 & $\begin{array}{c}267.12402\left([\mathrm{M}-\mathrm{H}-\mathrm{OH}]^{-}\right) \\
251.12881\left([\mathrm{M}-\mathrm{H}-2 \mathrm{OH}]^{-}\right) \\
235.13390\left([\mathrm{M}-\mathrm{H}-3 \mathrm{OH}]^{-}\right) \\
121.06506\left(\left[\mathrm{M}-\mathrm{H}-\mathrm{C}_{6} \mathrm{H}_{11} \mathrm{O}_{5}\right]^{-}\right)\end{array}$ & Root/shoot \\
\hline 43 & 12.75 & 272,368 & Dalbergioidin & $\mathrm{C}_{15} \mathrm{H}_{11} \mathrm{O}_{6}^{-}$ & 287.05611 & 287.05618 & 0.24 & $\begin{array}{c}271.06094\left([\mathrm{M}-\mathrm{H}-\mathrm{OH}]^{-}\right) \\
179.03467\left(\left[\mathrm{M}-\mathrm{H}_{-} \mathrm{C}_{6} \mathrm{H}_{5} \mathrm{O}_{2}\right]^{-}\right) \\
165.05252\left(\left[\mathrm{M}-\mathrm{H}^{-} \mathrm{C}_{6} \mathrm{H}_{5} \mathrm{O}_{3}\right]^{-}\right) \\
163.03951\left(\left[\mathrm{M}-\mathrm{H}-\mathrm{C}_{6} \mathrm{H}_{5} \mathrm{O}_{2}-\mathrm{OH}\right]^{-}\right) \\
147.04404\left(\left[\mathrm{M}-\mathrm{H}-\mathrm{C}_{6} \mathrm{H}_{5} \mathrm{O}_{2}-2 \mathrm{OH}\right]^{-}\right) \\
125.02380\left(\left[\mathrm{M}-\mathrm{H}-\mathrm{C}_{9} \mathrm{H}_{9} \mathrm{O}_{3}\right]^{-}\right) \\
109.02868\left(\left[\mathrm{M}-\mathrm{H}-\mathrm{C}_{9} \mathrm{H}_{7} \mathrm{O}_{4}\right]^{-}\right)\end{array}$ & Shoot \\
\hline 44 & 12.95 & 260 & 4,8,12-trihydroxy-2,4-dodecadienoic acid, & $\mathrm{C}_{12} \mathrm{H}_{19} \mathrm{O}_{5}^{-}$ & 243.1238 & 243.12383 & 0.12 & $\begin{array}{c}199.1337\left(\left[\mathrm{M}-\mathrm{H}-\mathrm{CHO}_{2}\right]^{-}\right) \\
139.11221\left(\left[\mathrm{M}-\mathrm{H}-\mathrm{CHO}_{2}-\mathrm{C}_{2} \mathrm{H}_{3}-2 \mathrm{OH}\right]^{-}\right)\end{array}$ & Root \\
\hline 45 & 13.40 & 283 & Caffeic acid isomer & $\mathrm{C}_{9} \mathrm{H}_{7} \mathrm{O}_{4}^{-}$ & 179.03498 & 179.03481 & 0.95 & $\begin{array}{c}163.03954\left([\mathrm{M}-\mathrm{H}-\mathrm{OH}]^{-}\right) \\
135.04454\left(\left[\mathrm{M}-\mathrm{H}-\mathrm{CHO}_{2}\right]^{-}\right) \\
109.02881\left(\left[\mathrm{M}-\mathrm{H}-\mathrm{C}_{3} \mathrm{H}_{3} \mathrm{O}_{2}\right]^{-}\right)\end{array}$ & Root \\
\hline 46 & 13.80 & 222,284 & $\begin{array}{c}\beta \text {-D-Glucopyranoside, } \\
\text { 1,1-dimethyl-5-methylenenonyl }\end{array}$ & $\mathrm{C}_{10} \mathrm{H}_{17} \mathrm{O}_{4}^{-}$ & 345.22826 & 345.22849 & 0.67 & $\begin{array}{c}327.21780\left([\mathrm{M}-\mathrm{H}-\mathrm{OH}]^{-}\right) \\
315.21780\left(\left[\mathrm{M}-\mathrm{H}-\mathrm{CH}_{3} \mathrm{O}\right]^{-}\right)\end{array}$ & Root \\
\hline
\end{tabular}


Table 1. Cont

\begin{tabular}{|c|c|c|c|c|c|c|c|c|c|}
\hline Peak & $\begin{array}{l}\text { Retention } \\
\text { Time (min) }\end{array}$ & $\begin{array}{l}\text { UVMax } \\
\left(\lambda_{\max }\right)\end{array}$ & Tentative Identification & $\begin{array}{c}\text { Elemental } \\
\text { Composition } \\
{[\mathrm{M}-\mathrm{H}]^{-}}\end{array}$ & $\begin{array}{l}\text { Theoretical } \\
\text { Mass }(m / z)\end{array}$ & $\begin{array}{l}\text { Measured } \\
\text { Mass } \\
(\mathrm{m} / \mathrm{z})\end{array}$ & $\begin{array}{l}\text { Accuracy } \\
\text { (ppm) }\end{array}$ & MSn Ions & Plant Part \\
\hline 47 & 13.90 & 224,284 & Sebacic acid & $\mathrm{C}_{10} \mathrm{H}_{17} \mathrm{O}_{4}^{-}$ & 201.11323 & 201.11293 & 1.49 & $\begin{array}{c}185.11778\left([\mathrm{M}-\mathrm{H}-\mathrm{OH}]^{-}\right) \\
157.12276\left(\left[\mathrm{M}-\mathrm{H}-\mathrm{CHO}_{2}\right]^{-}\right)\end{array}$ & Shoot \\
\hline 48 & 13.93 & 223,284 & alpha-Ionol O-[arabinosyl-(1->6)-glucoside] & $\mathrm{C}_{24} \mathrm{H}_{39} \mathrm{O}_{10}-$ & 487.25487 & 487.25504 & 0.35 & $\begin{array}{c}473.24008\left(\left[\mathrm{M}-\mathrm{H}-\mathrm{CH}_{3}\right]^{-}\right) \\
459.22311\left(\left[\mathrm{M}-\mathrm{H}-2 \mathrm{CH}_{3}\right]^{-}\right) \\
355.21292\left(\left[\mathrm{M}-\mathrm{H}-\mathrm{C}_{5} \mathrm{H}_{9} \mathrm{O}_{4}\right]^{-}\right) \\
341.19687\left(\left[\mathrm{M}-\mathrm{H}-\mathrm{C}_{5} \mathrm{H}_{9} \mathrm{O}_{4}-\mathrm{CH}_{3}\right]^{-}\right)\end{array}$ & Shoot \\
\hline 49 & 15.35 & 283,368 & Buteine & $\mathrm{C}_{15} \mathrm{H}_{11} \mathrm{O}_{5}^{-}$ & 271.0612 & 271.06131 & 0.41 & $\begin{array}{c}163.03952\left(\left[\mathrm{M}-\mathrm{H}-\mathrm{C}_{6} \mathrm{H}_{5} \mathrm{O}_{2}\right]^{-}\right) \\
137.02380\left(\left[\mathrm{M}-\mathrm{H}-\mathrm{C}_{8} \mathrm{H}_{7} \mathrm{O}_{2}\right]^{-}\right. \\
135.04443\left(\left[\mathrm{M}-\mathrm{H}-\mathrm{C}_{7} \mathrm{H}_{5} \mathrm{O}_{3}\right]^{-}\right) \\
121.02880\left(\left[\mathrm{M}-\mathrm{H}-\mathrm{C}_{8} \mathrm{H}_{7} \mathrm{O}_{2}-\mathrm{OH}\right]^{-}\right) \\
108.02104\left(\left[\mathrm{M}-\mathrm{H}-\mathrm{C}_{9} \mathrm{H}_{7} \mathrm{O}_{3}\right]^{-}\right)\end{array}$ & Root \\
\hline 50 & 15.98 & 283 & D-xylofuranose tetradecyl glycoside & $\mathrm{C}_{22} \mathrm{H}_{41} \mathrm{O}_{9}^{-}$ & 449.27561 & 449.27576 & 0.33 & $\begin{array}{c}403.27036\left(\left[\mathrm{M}-\mathrm{H}-\mathrm{CH}_{3} \mathrm{O}-\mathrm{OH}\right]^{-}\right) \\
316.22061\left(\left[\mathrm{M}-\mathrm{H}_{-}-\mathrm{C}_{5} \mathrm{H}_{9} \mathrm{O}_{4}\right]^{-}\right) \\
329.23349\left(\left[\mathrm{M}-\mathrm{H}_{-}-\mathrm{C}_{4} \mathrm{H}_{9}-4 \mathrm{OH}\right]^{-}\right) \\
117.05499\left(\left[\mathrm{M}-\mathrm{H}-\mathrm{C}_{17} \mathrm{H}_{32} \mathrm{O}_{5}-\mathrm{OH}\right]^{-}\right)\end{array}$ & Root \\
\hline 51 & 16.42 & 283 & Corchorifatty acid $\mathrm{F}$ isomer & $\mathrm{C}_{18} \mathrm{H}_{31} \mathrm{O}_{5}^{-}$ & 327.21770 & 327.21799 & 0.89 & $\begin{array}{c}309.20665\left([\mathrm{M}-\mathrm{H}-\mathrm{OH}]^{-}\right) \\
291.19684\left([\mathrm{M}-\mathrm{H}-2 \mathrm{OH}]^{-}\right) \\
173.11787\left(\left[\mathrm{M}-\mathrm{H}-\mathrm{C}_{9} \mathrm{H}_{15} \mathrm{O}_{2}\right]^{-}\right) \\
157.12346\left(\left[\mathrm{M}-\mathrm{H}-\mathrm{C}_{9} \mathrm{H}_{15} \mathrm{O}_{2}-\mathrm{OH}\right]^{-}\right) \\
125.09643\left(\left[\mathrm{M}-\mathrm{H}-\mathrm{C}_{3} \mathrm{H}_{5} \mathrm{O}_{2}-\mathrm{C}_{7} \mathrm{H}_{13} \mathrm{O}_{2}\right]^{-}\right)\end{array}$ & Root/shoot \\
\hline 52 & 18.30 & 283,368 & Tianshic acid & $\mathrm{C}_{18} \mathrm{H}_{33} \mathrm{O}_{5}^{-}$ & 329.23335 & 329.23361 & 0.79 & $\begin{array}{c}165.12788\left(\left[\mathrm{M}-\mathrm{H}-\mathrm{C}_{7} \mathrm{H}_{15} \mathrm{O}-3 \mathrm{OH}\right]^{-}\right) \\
127.11205\left(\left[\mathrm{M}-\mathrm{H}-\mathrm{C}_{10} \mathrm{H}_{19} \mathrm{O}_{2}-2 \mathrm{OH}\right]^{-}\right)\end{array}$ & Root/shoot \\
\hline 53 & 19.15 & 283 & Dimethyl sebacate (sebacic acid derivative) & $\mathrm{C}_{12} \mathrm{H}_{21} \mathrm{O}_{4}^{-}$ & 229.14453 & 229.144 & 0.39 & $\begin{array}{c}201.11287\left([\text { sebacic acid }]^{-}\right) \\
215.12865\left(\left[\mathrm{M}-\mathrm{H}-\mathrm{CH}_{3}\right]^{-}\right) \\
211.13374\left([\mathrm{M}-\mathrm{H}-\mathrm{O}]^{-}\right) \\
199.13379\left(\left[\mathrm{M}-\mathrm{H}-\mathrm{CH}_{3} \mathrm{O}\right]^{-}\right) \\
185.11778\left(\left[\mathrm{M}-\mathrm{H}-\mathrm{CH}_{3} \mathrm{O}-\mathrm{CH}_{3}\right]^{-}\right) \\
157.12303\left(\left[\mathrm{M}-\mathrm{H}-\mathrm{C}_{2} \mathrm{H}_{3} \mathrm{O}_{2}-\mathrm{CH}_{3}\right]^{-}\right) \\
\end{array}$ & Shoot \\
\hline 54 & 19.28 & 282,368 & Unknown & $\mathrm{C}_{13} \mathrm{H}_{27} \mathrm{O}_{8}{ }^{-}$ & - & 311.16888 & - & - & Root \\
\hline 55 & 19.47 & 283,368 & Corchorifatty acid $\mathrm{F}$ isomer & $\mathrm{C}_{18} \mathrm{H}_{31} \mathrm{O}_{5}^{-}$ & 327.21770 & 327.21802 & 0.98 & $\begin{array}{c}309.20731\left([\mathrm{M}-\mathrm{H}-\mathrm{OH}]^{-}\right) \\
291.19672\left([\mathrm{M}-\mathrm{H}-2 \mathrm{OH}]^{-}\right) \\
173.11792\left(\left[\mathrm{M}-\mathrm{H}-\mathrm{C}_{9} \mathrm{H}_{15} \mathrm{O}_{2}\right]^{-}\right) \\
125.09679\left(\left[\mathrm{M}-\mathrm{H}-\mathrm{C}_{3} \mathrm{H}_{5} \mathrm{O}_{2}-\mathrm{C}_{7} \mathrm{H}_{13} \mathrm{O}_{2}\right]^{-}\right)\end{array}$ & Root/shoot \\
\hline 56 & 20.06 & 283 & Unknown & $\mathrm{C}_{13} \mathrm{H}_{27} \mathrm{O}_{8}^{-}$ & - & 311.16888 & & - & Shoot \\
\hline
\end{tabular}


Table 1. Cont

\begin{tabular}{|c|c|c|c|c|c|c|c|c|c|}
\hline Peak & $\begin{array}{l}\text { Retention } \\
\text { Time (min) }\end{array}$ & $\begin{array}{l}\text { UVMax } \\
\left(\lambda_{\max }\right)\end{array}$ & Tentative Identification & $\begin{array}{c}\text { Elemental } \\
\text { Composition } \\
{[\mathrm{M}-\mathrm{H}]^{-}}\end{array}$ & $\begin{array}{l}\text { Theoretical } \\
\text { Mass }(m / z)\end{array}$ & $\begin{array}{l}\text { Measured } \\
\text { Mass } \\
(\mathrm{m} / \mathrm{z})\end{array}$ & $\begin{array}{c}\text { Accuracy } \\
\text { (ppm) }\end{array}$ & MSn Ions & Plant Part \\
\hline 57 & 20.63 & 283 & Nordihydrocapsiate & $\mathrm{C}_{17} \mathrm{H}_{25} \mathrm{O}_{4}^{-}$ & 293.17583 & 293.17612 & 0.99 & $\begin{array}{c}277.18088\left([\mathrm{M}-\mathrm{H}-\mathrm{OH}]^{-}\right) \\
263.16534\left(\left[\mathrm{M}-\mathrm{H}-\mathrm{CH}_{3} \mathrm{O}\right]^{-}\right) \\
247.16968\left(\left[\mathrm{M}-\mathrm{H}-\mathrm{CH}_{3} \mathrm{O}-\mathrm{OH}\right]^{-}\right) \\
157.12309\left(\left[\mathrm{M}-\mathrm{H}-\mathrm{C}_{8} \mathrm{H}_{9} \mathrm{O}_{2}\right]^{-}\right) \\
153.05524\left(\left[\mathrm{M}-\mathrm{H}-\mathrm{C}_{9} \mathrm{H}_{17} \mathrm{O}\right]^{-}\right) \\
141.12810\left(\left[\mathrm{M}-\mathrm{H}-\mathrm{C}_{8} \mathrm{H}_{9} \mathrm{O}_{3}\right]^{-}\right)\end{array}$ & Root/shoot \\
\hline 58 & 20.92 & 274 & Plastoquinone 3 & $\mathrm{C}_{23} \mathrm{H}_{31} \mathrm{O}_{2}^{-}$ & 339.23296 & 339.23322 & 0.77 & $\begin{array}{c}203.10753\left(\left[\mathrm{M}-\mathrm{H}-\mathrm{C}_{9} \mathrm{H}_{15}\right]^{-}\right) \\
163.11229\left(\left[\mathrm{M}-\mathrm{H}-\mathrm{C}_{6} \mathrm{H}_{11}-\mathrm{C}_{5} \mathrm{H}_{7} \mathrm{O}\right]^{-}\right) \\
149.06009\left(\left[\mathrm{M}-\mathrm{H}-\mathrm{C}_{13} \mathrm{H}_{21}\right]^{-}\right) \\
135.04454\left(\left[\mathrm{M}-\mathrm{H}-\mathrm{C}_{14} \mathrm{H}_{23}\right]^{-}\right)\end{array}$ & Root \\
\hline 59 & 21.25 & 283 & Decyl gallate (gallic acid derivative) & $\mathrm{C}_{17} \mathrm{H}_{25} \mathrm{O}_{5}^{-}$ & 309.17075 & 309.17093 & 0.58 & $\begin{array}{c}\left.293.17935 \text { ([M-H-OH] }]^{-}\right) \\
169.01381\left(\text { [gallic acid] }{ }^{-}\right) \\
153.01903\left(\left[\mathrm{M}-\mathrm{H}-\mathrm{C}_{10} \mathrm{H}_{21} \mathrm{O}\right]^{-}\right) \\
125.02367\left(\left[\mathrm{M}-\mathrm{H}-\mathrm{C}_{11} \mathrm{H}_{21} \mathrm{O}_{2}\right]^{-}\right)\end{array}$ & Root/shoot \\
\hline 60 & 21.61 & 283 & Nordihydrocapsiate isomer & $\mathrm{C}_{17} \mathrm{H}_{25} \mathrm{O}_{4}^{-}$ & 293.17583 & 293.17612 & 0.99 & $\begin{array}{c}277.18080\left([\mathrm{M}-\mathrm{H}-\mathrm{OH}]^{-}\right) \\
263.16535\left(\left[\mathrm{M}-\mathrm{H}-\mathrm{CH}_{3} \mathrm{O}\right]^{-}\right) \\
247.16990\left(\left[\mathrm{M}-\mathrm{H}-\mathrm{CH}_{3} \mathrm{O}-\mathrm{OH}\right]^{-}\right) \\
157.12309\left(\left[\mathrm{M}-\mathrm{H}-\mathrm{C}_{8} \mathrm{H}_{9} \mathrm{O}_{2}\right]^{-}\right) \\
153.05524\left(\left[\mathrm{M}-\mathrm{H}-\mathrm{C}_{9} \mathrm{H}_{17} \mathrm{O}\right]^{-}\right) \\
141.12810\left(\left[\mathrm{M}-\mathrm{H}-\mathrm{C}_{8} \mathrm{H}_{9} \mathrm{O}_{3}\right]^{-}\right)\end{array}$ & Root/shoot \\
\hline 61 & 22.30 & 283 & Unknown & $\mathrm{C}_{13} \mathrm{H}_{27} \mathrm{O}_{8}^{-}$ & - & 311.16904 & - & - & Root \\
\hline 62 & 22.53 & 283 & Unknown & $\mathrm{C}_{24} \mathrm{H}_{45} \mathrm{O}_{11}^{-}$ & - & 509.29691 & - & - & Shoot \\
\hline 63 & 23.36 & 283,337 & 13-Hydroxyoctadecadienoic acid & $\mathrm{C}_{18} \mathrm{H}_{31} \mathrm{O}_{3}^{-}$ & 295.22787 & 295.22797 & 0.34 & $\begin{array}{c}281.21204\left(\left[\mathrm{M}-\mathrm{H}-\mathrm{CH}_{3}\right]^{-}\right) \\
279.2333\left([\mathrm{M}-\mathrm{H}-\mathrm{OH}]^{-}\right) \\
169.12331\left(\left[\mathrm{M}-\mathrm{H}-\mathrm{C}_{8} \mathrm{H}_{15} \mathrm{O}\right]^{-}\right) \\
153.12767\left(\left[\mathrm{M}-\mathrm{H}-\mathrm{C}_{8} \mathrm{H}_{15} \mathrm{O}-\mathrm{OH}\right]^{-}\right)\end{array}$ & Root/shoot \\
\hline 64 & 23.54 & 283,337 & p-Hydroxynonanophenone & $\mathrm{C}_{15} \mathrm{H}_{21} \mathrm{O}_{2}^{-}$ & 233.1547 & 233.15462 & 0.34 & $\begin{array}{c}219.17544\left([\mathrm{M}-\mathrm{H}-\mathrm{O}]^{-}\right) \\
167.14342\left(\left[\mathrm{M}-\mathrm{H}-\mathrm{C}_{4} \mathrm{H}_{5} \mathrm{O}\right]^{-}\right) \\
135.04446\left(\left[\mathrm{M}-\mathrm{H}-\mathrm{C}_{7} \mathrm{H}_{15}\right]^{-}\right) \\
121.02875\left(\left[\mathrm{M}-\mathrm{H}-\mathrm{C}_{7} \mathrm{H}_{15}-\mathrm{CH}_{3}\right]^{-}\right)\end{array}$ & Root/shoot \\
\hline 65 & 24.03 & 283 & Unknown & $\mathrm{C}_{15} \mathrm{H}_{31} \mathrm{O}_{8}^{-}$ & - & 339.20029 & - & - & Root \\
\hline 66 & 25.20 & 283 & Unknown & $\mathrm{C}_{14} \mathrm{H}_{29} \mathrm{O}_{8}^{-}$ & - & 325.18463 & - & - & Root \\
\hline 67 & 25.72 & 283,337 & Unknown & $\mathrm{C}_{14} \mathrm{H}_{29} \mathrm{O}_{8}^{-}$ & - & 325.18457 & - & - & Shoot \\
\hline 68 & 25.98 & 283,337 & Unknown & $\mathrm{C}_{14} \mathrm{H}_{29} \mathrm{O}_{8}{ }^{-}$ & - & 325.18454 & - & - & Shoot \\
\hline 69 & 26.24 & 283 & Unknown & $\mathrm{C}_{14} \mathrm{H}_{29} \mathrm{O}_{8}^{-}$ & - & 325.18463 & - & - & Root \\
\hline
\end{tabular}


Compounds 1-5 were detected in both parts of $C$. macromeris. Compound $\mathbf{1}$ was assigned as vaccihein A (Figure 2a). This antioxidant metabolite was isolated for the first time in Vaccinium ashei (Ericaceae) by Ono, et al. [35] and this is the first time that is reported for cacti species. Compounds 2-5 were assigned as carboxylic acids: compounds $\mathbf{2}$ and $\mathbf{3}$ were proposed as dihydroxy methoxy butanoic acid and malic acid [36], respectively, and compounds $\mathbf{4}$ and $\mathbf{5}$ as isocitric acid isomers [13]. Citric acid occurs as one of the main hydrophilic constituents in Opuntia species (Cactaceae) [13,37], and its presence may be also related to the crassulacean acid metabolism of cacti species [12].

Among the detected compounds, 31 metabolites were phenolic acids (compounds 6-13, 15-25, 31, 32, 34, 36-42, 44, 48 and 62) and 16 of them occurred in the aerial part (see Table 1). In our investigation, compound 6 was assigned as 3,5-dihydroxy-4-methyloxolan-2-yl methoxy-6-hydroxymethyl oxane-3,4,5-triol, due to the presence of fragments ions at $\mathrm{m} / \mathrm{z}: 293.12424$, 279.10870, 147.06578, and 131.07106 (see Table 1); Vankudothu and Anwar [38] proposed, through molecular docking, that this metabolite showed high affinity for the ACC2 protein, suggesting an effect against type 2 diabetes. Thus, the elicitation and isolation of this compound may be a potential use for C. macromeris. Also, two protocatechuic acid derivatives (compounds 9 and 16) were detected: compound $\mathbf{9}$ was proposed as protocatechuic acid hexoside (see Figure $2 \mathrm{~b}$ ) and compound $\mathbf{1 6}$ as protocatechuic aldehyde since pseudomolecular ion at $m / z$ : 137.02386 yielded fragments at $m / z$ : 121.02882 and 109.02884 (see Table 1). It has been proposed that protocatechuic aldehyde influences the pharmacokinetic activity of medicinal herbal extracts prepared with Salvia miltiorrhiza (Lamiaceae) [39], suggesting that the presence of this compound in C. macromeris extract may enhance its functional properties. Protocatechuic acid has been found as an aglycone in other cacti species such as Opuntia ficus-indica [40] and Myrtillocactus geometrizans [17], and as one of the main constituents in Opuntia humifusa [18].

Additionally, compound $\mathbf{1 3}$ was only detected in the aerial part and was assigned as lucuminic acid, since the pseudomolecular ion yielded fragments at $m / z: 163.03947,119.04939$ and 107.04942 (see Table 1). Lucuminic acid has been reported for Calocarpum sapota (Sapotaceae) by Takeda, et al. [41] and this is the first time that is reported for cacti species. In our investigation, sinapic acid (compound 20) and two of its derivatives (compounds 19 and 25) were also detected and characterized as reported previously [42]. Sinapic acid is synthesized from ferulic acid through enzymatic mechanisms [43] and it has been proposed to have anti-inflammatory activities [44].

In the same way, other phenolic metabolites such as two syringic acid derivatives (compounds 21 and 23), two caffeic acid isomers [45] (compounds 24 and 45), and cinnamic and gallic acid derivatives (compound 31 and 59, respectively) were also found (see Table 1). In addition, piscidic and ferulic acid and/or their derivatives were detected as the most recurrent phenolic acids in C. macromeris extracts: six isomers of piscidic acid (compounds 7, 8, 10, 11, 12, and 15) and four if its derivatives (compounds 17, 18, 22, and 37) were detected and assigned as reported previously [46,47]. Piscidic acid has been previously identified in juices prepared with Opuntia ficus-indica fruits [13] and according to the number of peaks detected and the relative abundance shown in the chromatogram, piscidic acid may be one of the main constituents of the C. macromeris aerial part (see Figure 1a, Table 1); further studies are required to confirm the structure of this metabolite and its total concentration in each part of the plant. On the other hand, compounds $28,33,34$, and 36 were assigned as ferulic acid isomers [48], and compounds $29,35,38,39$, and 41 as ferulic acid derivatives, since they showed characteristic fragment ions of ferulic acid (see Table 1). Our results indicate that ferulic acid is present in both parts of the plant but with a higher relative abundance in the shoots (Figure 1a). Ferulic acid has been detected in different cacti species $[18,37,49,50]$ and its antiproliferative effect in human colon carcinoma HT29 cell line [51] as well its therapeutic potential against cardiovascular and neurodegenerative disorders [52] has been proposed. Our results suggest that $C$. macromeris could represent a potential source for the isolation of this compound or its derivatives; further studies are required to isolate and characterize the structure of these compounds (isomers) and to know their concentration, elicitation, and production in biotechnological systems. 
In addition to compounds $2-5$, compounds $26,27,32,40,44,47,51-53,55$, and 63 were also assigned as carboxylic acids. The fragmentation pattern of these metabolites was mainly characterized by the loss of water (see Table 1). Among these metabolites, compounds 51 and 55 were detected in both parts of the plant and assigned as corchorifatty acid $\mathrm{F}$ isomers. This metabolite has been previously identified in leaves of Corchorus olitorius (Tiliaceae) by Yoshikawa, et al. [53] and in aerial parts of Chaenomeles sinensis (Rosaceae) [54]. It also confers activity against pathogenic fungus Pyricularia oryzae in resistant rice cultivars [55]. To our knowledge, this is the first time that is reported for cacti species. On the other hand, compound $\mathbf{4 0}$ was identified as azelaic acid, according to Liu, et al. [56]. This dicarboxylic acid has been used for the treatment of acne and skin disorders $[57,58]$ and skin hyperpigmentation [59]. Compound $\mathbf{5 2}$ was assigned as tianshic acid due to the presence of fragments at $m / z: 165.12788$ and 127.11205 (see Table 1). The occurrence of this metabolite has been previously reported for Sambucus williamsii (Adoxaceae) by Yang, et al. [60] and no information exists for cacti species.

In the same way, other polar compounds were characterized in C. macromeris methanolic extracts. Compound $\mathbf{1 4}$ was assigned as hyrtioerectine $C$ (alkaloid). Youssef [61] isolated this alkaloid and proposed its cytotoxic activity against HeLa cells. In our research group, studies are being carried out to elucidate the presence of other alkaloids in, and the potential applications of, C. macromeris extracts. Signals revealed for compound 30 indicated the presence of a glucopyranoside derivative [62] in the aerial part of C. macromeris. Similarly, compound $\mathbf{4 2}$ was tentatively identified as 2-phenylethyl $\beta$-D-glucopyranoside. This phenolic glycoside has been previously reported in Pachysandra terminalis (Buxaceae) [63] and in Lactuca indica (Asteraceae) [64]. To our knowledge, this is the first time that it is reported for cacti species. Compound $\mathbf{4 3}$ was tentatively identified as dalbergioidin (see Figure 2d); this antifungal isoflavone was reported in Vigna angularis (Fabaceae) as one of the mechanisms against Phytophthora vignae zoospores germination [65], suggesting that its presence in the C. macromeris aerial part may correspond to a constitutive adaptation mechanism since no pathogenic conditions were given to the plants; further studies are required to assess this hypothesis. Compounds 46, 48, and 50 were also assigned as metabolites of glycosidic nature (see Table 1). On the other hand, the pseudomolecular ion and fragmentation pattern of compound 49 indicated the presence of buteine (chalcone; see Figure 2e) in the radicular part of C. macromeris. Buteine exists in Rhus verniciflua (Anacardiaceae), a traditional herb used for cancer treatment [66], and its antioxidant activity [67] and potential as a chemotherapeutic agent [68] has been proposed. Compounds 57 and $\mathbf{6 0}$ were tentatively identified as nordihidrocapsiate isomers (see Figure 2f) and compound 58 as plastoquinone 3.

Finally, compounds 54,56, 61, 62, and 65-69 were not identified since molecular information obtained did not match with theoretical information existing in the literature. Absorption spectrum of these compounds suggest that they may contain benzoic acid within its structure (UV $\lambda_{\max }: 280 \mathrm{~nm}$ ). Further studies are required for structural elucidation of these metabolites.
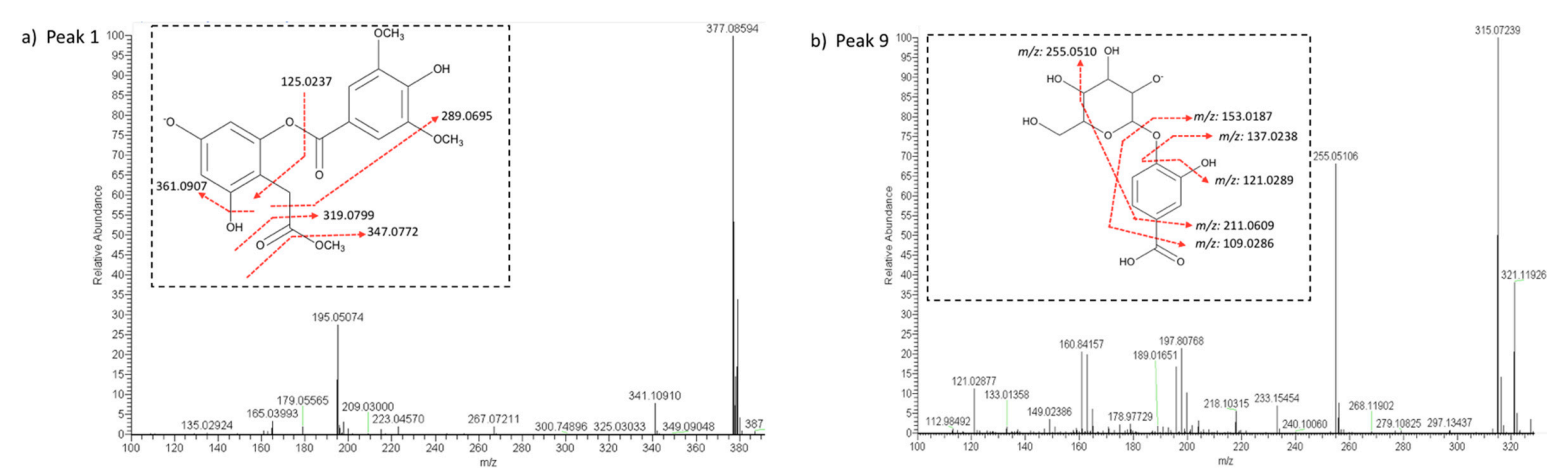

Figure 2. Cont. 

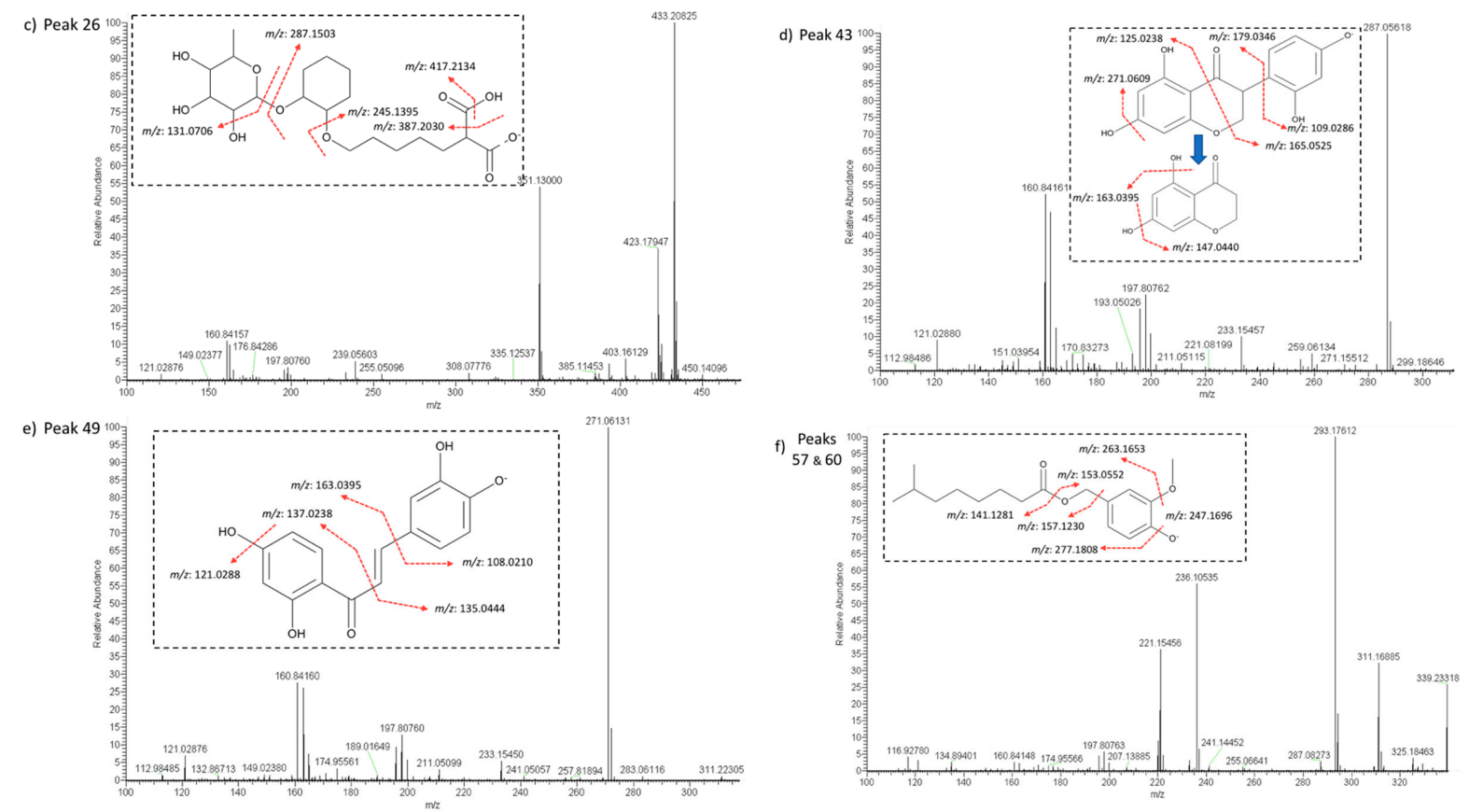

Figure 2. Full scan of some metabolites identified for the first time in C. macromeris. Dotted inset represents the molecule and red dotted lines in each inset represent the proposed fragmentation pattern. Peak numbers in each figure, refer to the compounds indicated in Table 1.

\section{Materials and Methods}

\subsection{Plant Material}

The plants of Coryphantha macromeris were obtained from the in vitro germplasm bank of the Autonomous University of Aguascalientes, México. Coryphantha macromeris was maintained on semisolid Murashige and Skoog medium [69] (3\% sucrose, $8 \mathrm{~g} \cdot \mathrm{L}^{-1}$ agar, and $\mathrm{pH}$ 5.7) and incubated at $25^{\circ} \mathrm{C}$ with fluorescent light $\left(40 \mu \mathrm{mol} \cdot \mathrm{m}^{2} \cdot \mathrm{s}^{-1}\right)$ and $16 / 8$ (light/dark) photoperiod. Each plant was subcultured for three months during one year with the described conditions, and then in vitro plants were acclimated to greenhouse conditions as reported previously [26] and kept for one year until harvesting for phytochemical analysis. The plant material was botanically identified by Professor Miguel Alvarado Rodríguez. A voucher specimen was deposited at the herbarium of the Autonomous University of Aguascalientes (HUAA; Voucher No. 6386).

\subsection{Sample Preparation}

For analysis, one-year-old plants growing under greenhouse conditions were collected and separated into aerial and radicular parts. Each part of the plant was sliced and dried in an oven $\left(40^{\circ} \mathrm{C}\right)$ during 1 week in dark conditions. Dried material was finally pulverized in a mortar and then extracted three times with methanol in an ultrasonic bath (30 min each time). The resultant extract was filtered and evaporated under reduced pressure at $40^{\circ} \mathrm{C}$ and freeze-dried (Labconco Freeze Dryer; Labconco Corporation, Kansas City, MO, USA). Each freeze-dried sample was resuspended $\left(2.5 \mathrm{mg} \cdot \mathrm{mL}^{-1}\right)$ in HPLC-MS-grade methanol and sonicated over $10 \mathrm{~min}$. All samples were filtered $(0.22 \mu \mathrm{m})$ and injected in an UHPLC system hyphenated with a mass spectrometer, as given below.

\subsection{UHPLC-PDA-HESI-Orbitrap-MS/MS Conditions}

Phytochemical analysis was performed as reported previously [32-34], using a Dionex Ultimate 3000 UHPLC system (Thermo Fisher Scientific, Bremen, Germany) with a C18 column (ID: $150 \times 4.6 \mathrm{~mm}, 5 \mu \mathrm{m}$; Restek Corporation, Bellefonte, PA, USA) and equipped with a Quaternary Series RS pump and a Dionex Ultimate 3000 Series TCC-3000RS column compartments with an 
Ultimate 3000 Series WPS-3000RS autosampler (Thermo Fisher Scientific) and a rapid separations PDA detector. The detection wavelengths were 254, 280, 320, and $440 \mathrm{~nm}$, and PDA was recorded from 200 to $800 \mathrm{~nm}$ for peak characterization. The separation was performed in a gradient elution mode composed by $1 \%$ formic aqueous solution (A) and acetonitrile (B). The flow rate was $1.0 \mathrm{~mL} \cdot \mathrm{min}^{-1}$ and the injection volume $10 \mu \mathrm{L}$. The gradient program [time $(\mathrm{min}), \% \mathrm{~B}$ ] was: $(0.00,5),(5.00,5),(10.00,30)$, $(15.00,30),(20.00,70),(25.00,70),(35.00,5)$, and $12 \mathrm{~min}$ for column equilibration before each injection. The system was controlled by Chromeleon 7.2 Software (Thermo Fisher Scientific, Waltham, MA, USA and Dionex Softron GmbH division of Thermo Fisher Scientific) and hyphenated with a Thermo high resolution Q Exactive focus mass spectrometer (Thermo Fisher Scientific). The chromatographic system was coupled to the mass spectrometer with a heated electrospray ionization source II (HESI II). Nitrogen (purity $>99.999 \%$ ) was employed as both the collision and damping gas. Nitrogen was obtained from a Genius NM32LA nitrogen generator (Peak Scientific, Billerica, MA, USA). Mass calibration for Orbitrap was performed once a week, in both negative and positive modes. Caffeine and N-butylamine (Sigma-Aldrich, Saint Louis, MO, USA) were the calibration standards for positive ions and buspirone hydrochloride, sodium dodecyl sulfate, and taurocholic acid sodium salt were used to calibrate the mass spectrometer. These compounds were dissolved in a mixture of acetic acid, acetonitrile, water, and methanol (Merck Darmstadt, Hesse, Germany) and infused using a Chemyx Fusion 100 syringe pump. XCalibur 2.3 software and Trace Finder 3.2 (Thermo Fisher Scientific, San Jose, CA, USA) were used for UHPLC control and data processing, respectively. Q Exactive 2.0 SP 2 (Thermo Fisher Scientific, Waltham, MA, USA) was used to control the mass spectrometer.

\section{MS Parameters}

The HESI parameters were optimized as follows: sheath gas flow rate 75 units; auxiliary gas flow rate 20 units; capillary temperature $400{ }^{\circ} \mathrm{C}$; auxiliary gas heater temperature $500{ }^{\circ} \mathrm{C}$; spray voltage $2500 \mathrm{~V}$ (for ESI-); and S lens RF level 30. Full scan data in negative mode was acquired at a resolving power of 70,000 full width half maximum (FWHM) at $m / z 200$. For the compounds of interest, a scan range of $m / z 100-1000$ was chosen; the automatic gain control (AGC) was set at $3 \times 10^{6}$ and the injection time was set to $200 \mathrm{~ms}$. Scan rate was set at $2 \mathrm{scans} \mathrm{s}^{-1}$. External calibration was performed using a calibration solution in positive and negative modes before each sample series. In addition to the full scan acquisition method, for confirmation purposes, a targeted MS/MS analysis was performed using the mass inclusion list and expected retention times of the target analytes, with a $30 \mathrm{~s}$ time window, with the Orbitrap spectrometer operating both in positive and negative mode at 17,500 FWHM $(m / z 200)$. The AGC target was set to $2 \times 10^{5}$, with the maximum injection time of $20 \mathrm{~ms}$. The precursor ions were filtered by the quadrupole operating at an isolation window of $m / z 2$. The fore vacuum, high vacuum, and ultra-high vacuum were maintained at approximately 2 mbar, from 105 to below 1010 mbar, respectively. Collision energy (HCD cell) was operated at $30 \mathrm{eV}$. Detection was based on calculated exact mass and on retention time of target compounds presented in Table 1 . The mass tolerance window was set to $5 \mathrm{ppm}$.

\section{Conclusions}

The utilized procedures allowed the separation of 69 metabolites present in methanolic extracts of aerial and radicular parts of C. macromeris, and the identification of most of them. The aerial part showed higher diversity of metabolites compared with roots, and 24 metabolites occurred in both plant parts. Among detected compounds, ferulic and piscidic acid and their derivatives were the most recurrent phenolic metabolites in C. macromeris. In addition, different classes of compounds with reported functional properties were detected. To our knowledge, the phytochemical profile of aerial and root parts of $C$. macromeris is reported here for the first time by means of modern ultra-high-performance liquid chromatography-tandem mass spectrometry. Based on the detected compounds, C. macromeris may find potential applications in the pharmaceutical and cosmetic industries. The obtained information could be also useful as chemotaxonomic markers for Coryphantha species. 
Author Contributions: Data curation, J.J.-R.; formal analysis, F.C.-S.; funding acquisition, E.P.-M.B.; investigation, E.C.-G.; methodology, C.A. Additionally: E.C.-G. contributed to the design of the project leading to this publication, performed the experiments, analyzed results, and wrote the manuscript; E.P.-M.B. and F.C.-S. designed and directed the project leading to this publication, critically reviewed the manuscript for important intellectual content, and gave financial support for the project leading to this publication; C.A. directed the chemical analysis, analyzed results and critically reviewed the manuscript for important intellectual content; J.J.-R. critically reviewed the manuscript and contributed to the planning and execution of the project leading to this publication. All authors helped with the preparation of the manuscript and approved the final version.

Funding: This research was funded by the National Council for Science and Technology (CONACYT), grant number 366290, and resources given by the Autonomous University of Aguascalientes and by the National Fund of Scientific and Technological Development of Chile (Fondecyt No. 1150745).

Acknowledgments: We thank Ruben Muñoz and Jorge Bórquez Ramirez from the University of Antofagasta, Chile, for technical support.

Conflicts of Interest: The authors declare no conflict of interest.

\section{References}

1. Santos-Díaz, M.S.; Pérez-Molphe, E.; Ramírez-Malagón, R.; Núñez-Palenius, H.G.; Ochoa- Alejo, N. Mexican Threatened Cacti: Current Status and Strategies for Their Conservation. In Species Diversity and Extinction; Tepper, G.H., Ed.; Nova Science Publishers, Inc.: Hauppauge, NY, USA, 2010; pp. 1-60.

2. Mauseth, J.D. Biogeography and biodiversity of cacti. Cactus Succul. J. 2016, 88, 46. [CrossRef]

3. Martins, C.; Oliveira, R.; Mendonça Filho, C.V.; Teixeira Lopes, L.; Assunção Silveira, R.; Pereira de Silva, J.A.; Aguiar, L.M.S.; Antonini, Y. Reproductive biology of Cipocereus minensis (Cactaceae)—A columnar cactus endemic to rupestrian fields of a Neotropical savannah. Flora 2016, 218, 62-67. [CrossRef]

4. Saenz, C.; Sepúlveda, E.; Matsuhiro, B. Opuntia spp. mucilage's: A functional component with industrial perspectives. J. Arid Environ. 2004, 57, 275-290. [CrossRef]

5. Matsuhiro, B.; Lillo, L.E.; Sáenz, C.; Urzúa, C.C.; Zárate, O. Chemical characterization of the mucilage from fruits of Opuntia ficus indica. Carbohydr. Polym. 2006, 63, 263-267. [CrossRef]

6. Sepúlveda, E.; Sáenz, C.; Aliaga, E.; Aceituno, C. Extraction and characterization of mucilage in Opuntia spp. J. Arid Environ. 2007, 68, 534-545. [CrossRef]

7. Valente, L.M.M.; da Paixão, D.; do Nascimento, A.C.; dos Santos, P.F.P.; Scheinvar, L.A.; Moura, M.R.L.; Tinoco, L.W.; Gomes, L.N.F.; da Silva, J.F.M. Antiradical activity, nutritional potential and flavonoids of the cladodes of Opuntia monacantha (Cactaceae). Food Chem. 2010, 123, 1127-1131. [CrossRef]

8. Zampini, I.C.; Ordoñez, R.; Giannini, N.P.; Blendinger, P.G.; Isla, M.I. Nutraceutical properties and toxicity studies of fruits from four Cactaceae species grown in Argentine Northwestern. Food Res. Int. 2011, 44, 2345-2351. [CrossRef]

9. Ammar, I.; Ennouri, M.; Khemakhem, B.; Yangui, T.; Attia, H. Variation in chemical composition and biological activities of two species of Opuntia flowers at four stages of flowering. Ind. Crops Prod. 2012, 37, 34-40. [CrossRef]

10. Starha, R.; Chybidziurová, A.; Lance, Z. Alkaloids of the genus Turbinicarpus (Cactaceae). Biochem. Syst. Ecol. 1999, 27, 839-841. [CrossRef]

11. Ogunbodede, O.; McCombs, D.; Trout, K.; Daley, P.; Terry, M. New mescaline concentrations from 14 taxa/cultivars of Echinopsis spp. (Cactaceae) ("San Pedro") and their relevance to shamanic practice. J. Ethnopharmacol. 2010, 131, 356-362. [CrossRef] [PubMed]

12. Astello-García, M.G.; Cervantes, I.; Nair, V.; Santos-Díaz, M.d.S.; Reyes-Agüero, A.; Guéraud, F.; Negre-Salvayre, A.; Rossignol, M.; Cisneros-Zevallos, L.; Barba de la Rosa, A.P. Chemical composition and phenolic compounds profile of cladodes from Opuntia spp. cultivars with different domestication gradient. J. Food Compos. Anal. 2015, 43, 119-130. [CrossRef]

13. Mata, A.; Ferreira, J.P.; Semedo, C.; Serra, T.; Duarte, C.M.M.; Bronze, M.R. Contribution to the characterization of Opuntia spp. juices by LC-DAD-ESI-MS/MS. Food Chem. 2016, 210, 558-565. [CrossRef] [PubMed]

14. Ammar, I.; Ennouri, M.; Attia, H. Phenolic content and antioxidant activity of cactus (Opuntia ficus-indica L.) flowers are modified according to the extraction method. Ind. Crops Prod. 2015, 64, 97-104. [CrossRef] 
15. Mokrani, A.; Madani, K. Effect of solvent, time and temperature on the extraction of phenolic compounds and antioxidant capacity of peach (Prunus persica L.) fruit. Sep. Purif. Technol. 2016, 162, 68-76. [CrossRef]

16. Saïdani Tounsi, M.; Ouerghemmi, I.; Ksouri, R.; Aidi Wannes, W.; Hammrouni, I.; Marzouk, B. HPLC-determination of phenolic composition and antioxidant capacity of cactus prickly pears seeds. Asian J. Chem. 2011, 23, 1006-1010.

17. Herrera-Hernández, M.G.; Guevara-Lara, F.; Reynoso-Camacho, R.; Guzmán-Maldonado, S.H. Effects of maturity stage and storage on cactus berry (Myrtillocactus geometrizans) phenolics, vitamin C, betalains and their antioxidant properties. Food Chem. 2011, 129, 1744-1750. [CrossRef]

18. Cha, M.-N.; Jun, H.-I.; Lee, W.-J.; Kim, M.-J.; Kim, M.-K.; Kim, Y.-S. Chemical composition and antioxidant activity of Korean cactus (Opuntia humifusa) fruit. Food Sci. Biotechnol. 2013, 22, 523-529. [CrossRef]

19. Jun, H.-I.; Cha, M.-N.; Yang, E.-I.; Choi, D.G.; Kim, Y.-S. Physicochemical properties and antioxidant activity of Korean cactus (Opuntia humifusa) cladodes. Hortic. Environ. Biotechnol. 2013, 54, 288-295. [CrossRef]

20. Werner, E.; Heilier, J.-F.; Ducruix, C.; Ezan, E.; Junot, C.; Tabet, J.-C. Mass spectrometry for the identification of the discriminating signals from metabolomics: Current status and future trends. J. Chromatogr. B 2008, 871, 143-163. [CrossRef]

21. Lucci, P.; Saurina, J.; Núñez, O. Trends in LC-MS and LC-HRMS analysis and characterization of polyphenols in food. TrAC Trends Anal. Chem. 2017, 88, 1-24. [CrossRef]

22. Castro-Puyana, M.; Pérez-Míguez, R.; Montero, L.; Herrero, M. Application of mass spectrometry-based metabolomics approaches for food safety, quality and traceability. TrAC Trends Anal. Chem. 2017, 93, 102-118. [CrossRef]

23. Veeresham, C. Natural products derived from plants as a source of drugs. J. Adv. Pharm. Technol. Res. 2012, 3, 200-201. [CrossRef] [PubMed]

24. Ram, S. Chemotaxonomy: A tool for plant classification. J. Med. Plants Stud. 2016, 4, 90-93.

25. Dicht, R.; Lüthy, A. Coryphantha-Cacti of Mexico and Southern USA; Springer: Berlin/Heidelberg, Germany, 2005; pp. 3-5. [CrossRef]

26. Pérez-Molphe-Balch, E.; Pérez-Reyes, M.E.; Dávila-Figueroa, C.A.; Villalobos-Amador, E. In vitro propagation of three species of columnar cacti from the Sonoran desert. HortScience 2002, 37, 693-696. [CrossRef]

27. Pérez-Molphe-Balch, E.; Santos-Díaz, M.d.S.; Ramírez-Malagón, R.; Ochoa-Alejo, N. Tissue culture of ornamental cacti. Sci. Agric. 2015, 72, 540-561. [CrossRef]

28. Batis, A.; Rojas, M. El peyote y otros cactos alucinógenos de México. CONABIO Biodiversitas 2002, 40, $12-17$.

29. Keller, W.J.; McLaughlin, J.L. Cactus Alkaloids XIII: Isolation of (-)—Normacromerine from Coryphantha macromeris var. runyonii. J. Pharm. Sci. 1972, 61, 147-148. [CrossRef]

30. Kikuchi, H.; Uchiyama, N.; Ogata, J.; Kikura-Hanajiri, R.; Goda, Y. Chemical constituents and DNA sequence analysis of a psychotropic herbal product. Forensic Toxicol. 2010, 28,77-83. [CrossRef]

31. La Barbera, G.; Capriotti, A.L.; Cavaliere, C.; Montone, C.M.; Piovesana, S.; Samperi, R.; Zenezini Chiozzi, R.; Laganà, A. Liquid chromatography-high resolution mass spectrometry for the analysis of phytochemicals in vegetal-derived food and beverages. Food Res. Int. 2017, 100, 28-52. [CrossRef]

32. Cornejo, A.; Salgado, F.; Caballero, J.; Vargas, R.; Simirgiotis, M.; Areche, C. Secondary metabolites in Ramalina terebrata detected by UHPLC/ESI/MS/MS and identification of parietin as Tau protein inhibitor. Int. J. Mol. Sci. 2016, 17, 1303. [CrossRef]

33. Simirgiotis, M.; Quispe, C.; Borquez, J.; Areche, C.; Sepulveda, B. Fast detection of phenolic compounds in extracts of easter pears (Pyrus communis) from the Atacama desert by Ultra High-Performance Liquid Chromatography and Mass Spectrometry (UHPLC-Q/Orbitrap/MS/MS). Molecules 2016, 21, 92. [CrossRef]

34. Simirgiotis, M.; Quispe, C.; Areche, C.; Sepulveda, B. Phenolic compounds in Chilean Mistletoe (Quintral, Tristerix tetrandus) analyzed by UHPLC-Q/Orbitrap/MS/MS and its antioxidant properties. Molecules 2016, 21, 245. [CrossRef] [PubMed]

35. Ono, M.; Masuoka, C.; Koto, M.; Tateishi, M.; Komatsu, H.; Kobayashi, H.; Igoshi, K.; Ito, Y.; Okawa, M.; Nohara, T. Antioxidant ortho-benzoyloxyphenyl acetic acid ester, vaccihein A, from the fruit of rabbiteye blueberry (Vaccinium ashei). Chem. Pharm. Bull. 2002, 50, 1416-1417. [CrossRef] [PubMed]

36. Ledesma-Escobar, C.A.; Priego-Capote, F.; Luque de Castro, M.D. Characterization of lemon (Citrus limon) polar extract by liquid chromatography-tandem mass spectrometry in high resolution mode. J. Mass Spectrom. 2015, 50, 1196-1205. [CrossRef] [PubMed] 
37. Morales, P.; Barros, L.; Ramírez-Moreno, E.; Santos-Buelga, C.; Ferreira, I.C.F.R. Exploring xoconostle by-products as sources of bioactive compounds. Food Res. Int. 2014, 65(Part C), 437-444. [CrossRef]

38. Vankudothu, N.; Anwar, S.Y. Molecular interaction studies of Thespesia populnea extracts and their analogs on therapeutic targets by molecular docking. Int. J. Pharm. Sci. Rev. Res. 2014, 29, 82-85.

39. Chang, B.-B.; Zhang, L.; Cao, W.-W.; Cao, Y.; Yang, W.-L.; Wang, Y.; Chen, Y.-C.; Liu, X.-Q. Pharmacokinetic interactions induced by content variation of major water-soluble components of Danshen preparation in rats. Acta Pharmacol. Sin. 2010, 31, 638-646. [CrossRef]

40. Allai, L.; Karym, E.M.; El Amiri, B.; Nasser, B.; Essamad, A.; Terzioğlu, P.; Ertas, A.; Öztürk, M. Evaluation of antioxidant activity and phenolic composition of Opuntia ficus-indica cladodes collected from Moroccan Settat region. Eurasian J. Anal. Chem. 2016, 12, 105-117. [CrossRef]

41. Takeda, T.; Gonda, R.; Hatano, K. Constitution of Lucumin and its related glycosides from Calocarpum sapota Merrill. Chem. Pharm. Bull. 1997, 45, 697-699. [CrossRef]

42. Martínez-Las Heras, R.; Quifer-Rada, P.; Andrés, A.; Lamuela-Raventós, R. Polyphenolic profile of persimmon leaves by high resolution mass spectrometry (LC-ESI-LTQ-Orbitrap-MS). J. Funct. Food. 2016, 23, 370-377. [CrossRef]

43. Heleno, S.A.; Martins, A.; Queiroz, M.J.R.P.; Ferreira, I.C.F.R. Bioactivity of phenolic acids: Metabolites versus parent compounds: A review. Food Chem. 2015, 173, 501-513. [CrossRef] [PubMed]

44. Yun, K.-J.; Koh, D.-J.; Kim, S.-H.; Park, S.J.; Ryu, J.H.; Kim, D.-G.; Lee, J.-Y.; Lee, K.-T. Anti-Inflammatory effects of sinapic acid through the suppression of inducible nitric oxide synthase, cyclooxygase-2, and proinflammatory cytokines expressions via nuclear factor-kappaB inactivation. J. Agric. Food. Chem. 2008, 56, 10265-10272. [CrossRef] [PubMed]

45. Ożarowski, M.; Piasecka, A.; Gryszczyńska, A.; Sawikowska, A.; Pietrowiak, A.; Opala, B.; Mikołajczak, P.Ł.; Kujawski, R.; Kachlicki, P.; Buchwald, W.; et al. Determination of phenolic compounds and diterpenes in roots of Salvia miltiorrhiza and Salvia przewalskii by two LC-MS tools: Multi-stage and high resolution tandem mass spectrometry with assessment of antioxidant capacity. Phytochem. Lett. 2017, 20, 331-338. [CrossRef]

46. Maier, C.; Conrad, J.; Carle, R.; Weiss, J.; Schweiggert, R.M. Phenolic constituents in commercial aqueous Quillaja (Quillaja saponaria Molina) wood extracts. J. Agric. Food. Chem. 2015, 63, 1756-1762. [CrossRef] [PubMed]

47. Fan, M.; Qin, K.; Ding, F.; Huang, Y.; Wang, X.; Cai, B. Identification and differentiation of major components in three different "Sheng-ma" crude drug species by UPLC/Q-TOF-MS. Acta Pharm. Sin. B 2017, 7, 185-192. [CrossRef] [PubMed]

48. Sulaiman, C.T.; Nasiya, K.K.; Balachandran, I. Isolation and mass spectroscopic characterization of phytochemicals from the bark of Acacia leucophloea (Roxb.) Willd. Spectrosc. Lett. 2016, 49, 391-395. [CrossRef]

49. Benayad, Z.; Martinez-Villaluenga, C.; Frias, J.; Gomez-Cordoves, C.; Es-Safi, N.E. Phenolic composition, antioxidant and anti-inflammatory activities of extracts from Moroccan Opuntia ficus-indica flowers obtained by different extraction methods. Ind. Crops Prod. 2014, 62, 412-420. [CrossRef]

50. Jiménez-Aspee, F.; Quispe, C.; Camarantin Soriano, M.d.P.; Fuentes Gonzalez, J.; Hüneke, E.; Theoduloz, C.; Schmeda-Hirschmann, G. Antioxidant activity and characterization of constituents in copao fruits (Eulychnia acida Phil., Cactaceae) by HPLC-DAD-MS/MSn. Food Res. Int. 2014, 62, 286-298. [CrossRef]

51. Serra, A.T.; Poejo, J.; Matias, A.A.; Bronze, M.R.; Duarte, C.M.M. Evaluation of Opuntia spp. derived products as antiproliferative agents in human colon cancer cell line (HT29). Food Res. Int. 2013, 54, 892-901. [CrossRef]

52. Srinivasan, M.; Sudheer, A.R.; Menon, V.P. Ferulic acid: Therapeutic potential through its antioxidant property. J. Clin. Biochem. Nutr. 2007, 40, 92-100. [CrossRef]

53. Yoshikawa, M.; Murakami, T.; Shimada, H.; Yoshizumi, S.; Saka, M.; Yamahara, J.; Matsuda, H. Medicinal foodstuffs. XIV. On the bioactive constituents of Moroheiya. (2): New fatty Acids, Corchorifatty Acids A, B, C, D, E, and F, from the leaves of Corchorus olitorius L. (Tiliaceae): Structures and inhibitory effect on NO production in mouse peritoneal macrophages. Chem. Pharm. Bull. 1998, 46, 1008-1014. [PubMed]

54. Kim, C.S.; Kwon, O.W.; Kim, S.Y.; Choi, S.U.; Kim, K.H.; Lee, K.R. Five new oxylipins from Chaenomeles sinensis. Lipids 2014, 49, 1151-1159. [CrossRef] [PubMed]

55. Kato, T.; Yamaguchi, Y.; Abe, N.; Uyehara, T.; Namai, T.; Kodama, M.; Shiobara, Y. Structure and synthesis of unsaturaded trihydroxy C18 fatty: Acids in rice plants suffering from rice blast disease. Tetrahedron Lett. 1985, 26, 2357-2360. [CrossRef] 
56. Liu, M.; Li, P.; Zeng, X.; Wu, H.; Su, W.; He, J. Identification and pharmacokinetics of multiple potential bioactive constituents after oral administration of Radix Astragali on cyclophosphamide-induced immunosuppression in Balb/c mice. Int. J. Mol. Sci. 2015, 16, 5047-5071. [CrossRef] [PubMed]

57. Burchacka, E.; Potaczek, P.; Paduszyński, P.; Karłowicz-Bodalska, K.; Han, T.; Han, S. New effective azelaic acid liposomal gel formulation of enhanced pharmaceutical bioavailability. Biomed. Pharmacother. 2016, 83, 771-775. [CrossRef] [PubMed]

58. Mayer-da-Silva, A.; Gollnick, H.; Detmar, M.; Gassmuller, J.; Parry, A.; Muller, R.; Orfanos, C.E. Effects of azelaic acid on sebaceous gland, sebum excretion rate and keratinization pattern in human skin. An in vivo and in vitro study. Acta Dermato Venereol. Suppl. 1989, 143, 20-30.

59. Lowe, N.J.; Rizk, D.; Grimes, P.; Billips, M.; Pincus, S. Azelaic acid 20\% cream in the treatment of facial hyperpigmentation in darker-skinned patients. Clin. Ther. 1998, 20, 945-959. [CrossRef]

60. Yang, X.; Wong, M.; Wang, N.; Chan, A.S.; Yao, X. A new eudesmane derivative and a new fatty acid ester from Sambucus williamsii. Chem. Pharm. Bull. 2006, 54, 676-678. [CrossRef]

61. Youssef, D.T. Hyrtioerectines A-C, cytotoxic alkaloids from the red sea sponge Hyrtios erectus. J. Nat. Prod. 2005, 68, 1416-1419. [CrossRef]

62. Li, H.; Yu, Y.; Wang, Z.; Geng, J.; Dai, Y.; Xiao, W.; Yao, X. Chemical profiling of Re-Du-Ning injection by ultra-performance liquid chromatography coupled with electrospray ionization tandem quadrupole time-of-flight mass spectrometry through the screening of diagnostic ions in MS(E) mode. PLoS ONE 2015, 10, e0121031. [CrossRef]

63. Li, C.Y.; Zhai, H.Y.; Tang, S.A.; Duan, H.Q. Studies on the chemical constituents of Pachysandra terminalis and their antioxidant activity. Zhong Yao Cai 2010, 33, 729-732. [PubMed]

64. Kim, K.H.; Lee, K.H.; Choi, S.U.; Kim, Y.H.; Lee, K.R. Terpene and phenolic constituents of Lactuca indica L. Arch. Pharm. Res. 2008, 31, 983-988. [CrossRef] [PubMed]

65. Harada, G.; Kondo, N. Induction of phytoalexins in adzuki bean after inoculation with Phytophthora vignae $\mathrm{f}$. sp. adzukicola. J. Gen. Plant Pathol. 2009, 75, 432-436. [CrossRef]

66. Samoszuk, M.; Tan, J.; Chorn, G. The chalcone butein from Rhus verniciflua Stokes inhibits clonogenic growth of human breast cancer cells co-cultured with fibroblasts. BMC Complement. Altern. Med. 2005, 5, 5. [CrossRef] [PubMed]

67. Cheng, Z.-J.; Kuo, S.-C.; Chan, S.-C.; Ko, F.-N.; Teng, C.-M. Antioxidant properties of butein isolated from Dalbergia odorifera. Biochim. Biophys. Acta-Lipids Lipid Metab. 1998, 1392, 291-299. [CrossRef]

68. Jayasooriya, R.G.P.T.; Molagoda, I.M.N.; Park, C.; Jeong, J.-W.; Choi, Y.H.; Moon, D.-O.; Kim, M.-O.; Kim, G.-Y. Molecular chemotherapeutic potential of butein: A concise review. Food Chem. Toxicol. 2018, 112, 1-10. [CrossRef] [PubMed]

69. Murashige, T.; Skoog, F. A revised medium for rapid growth and bio assays with Tobacco tissue cultures. Physiologia Plantarum 1962, 15, 473-497. [CrossRef]

Sample Availability: Samples of plant material and extracs are available from the authors.

(C) 2019 by the authors. Licensee MDPI, Basel, Switzerland. This article is an open access article distributed under the terms and conditions of the Creative Commons Attribution (CC BY) license (http:/ / creativecommons.org/licenses/by/4.0/). 